Pacific Journal of Mathematics

MAPPINGS AND SPACES 


\title{
MAPPINGS AND SPACES
}

\author{
TAKESI ISIWATA
}

Let $\varphi$ be a closed continuous mapping from $X$ onto $Y$. It is an open problem whether the realcompactness of $X$ implies the realcompactness of $Y$. Concerning this problem, in case $\varphi$ is an open WZ-mapping, we discuss the structure of the image space $Y$ under $\varphi$ and give a necessary and sufficient condition that $Y$ be realcompact. We also show that if $X$ is locally compact, countably paracompact, normal space then the image space $Y$ of $X$ under a closed mapping is realcompact when $X$ is realcompact.

The notion of realcompact space was introduced by E. Hewitt [7] under the name of $Q$-spaces. The importance of this notion has been recognized and investigated by many mathematicians (cf. [4, 7]). In this paper we shall discuss the relations between realcompactness and closed continuous mappings and treat also the relations between pseudocompactness and continuous mappings.

As a generalization of closed mappings ${ }^{1}$, we have a $Z$-mapping. Here we shall introduce the notion of $W Z$-mappings as a further generalization of closed mappings. In Theorem 2.1, we shall prove that pseudocompactness of a space $X$ is equivalent to any one of the following conditions: 1) any continuous mapping from $X$ onto any weakly separable space is always a $Z$-mapping, (2) the projection: $Y \times X \rightarrow Y$ is a $Z$-mapping for any weakly separable space $Y$. We denote by $\varphi: X \rightarrow Y$ a mapping $\varphi$ from $X$ onto $Y$; then $\varphi$ can be extended to a continuous mapping $\Phi: \beta X \rightarrow \beta Y$, called the Stone extension of $\varphi$, where $\beta X$ and $\beta Y$ are the Stone Čech compactifications of $X$ and $Y$ resp. (In the sequel we denote always by $\Phi$ the Stone extension of $\varphi$ ). In $\S 4$, we shall deal with an extension of an open mapping, and show, in Theorem 4.4, that if $\varphi: X \rightarrow Y$ is a $W Z$ mapping, then $\Phi$ is open if and only if $\varphi$ is open. This plays an important role in $\S 6$. We shall consider in $\S 5$ the inverse images of realcompact space under $Z$-mappings. It is known that if $\varphi$ is a mapping from a given space $X$ onto a realcompact space $Y$, then $\Phi^{-1}(Y)$ is realcompact [4, p. 148]. In Theorem 5.3, we shall show

1 Throughout this paper we assume that all our spaces are completely regular $T_{1}$-spaces and mappings are continuous. We use, in the sequel. the same notations as in [4]. For instance, $C(X)$ is the set of all continuous functions defined on $X$. A subset $F$ of $X$ is said to be a zero set if $F=\left\{x ; f(x)=0\right.$ \} (briefly, $F=Z(f)=Z_{X}(f)$ ) for some $f \in C(X)$. $\quad \mathrm{C}_{A}$ denotes a closure operation in a space $A$. 
that if $\varphi$ is a $Z$-mapping from a space $X$ onto a realcompact space $Y$ such that every $\varphi^{-1}(y), y \in Y$, is a $C^{*}$-embedded realcompact subset of $X$, then $X$ is realcompact. In particular, if $X$ is normal and every $\varphi^{-1}(y), y \in Y$, is realcompact, then realcompactness is invariant under $\varphi^{-1}$.

It is an open problem [4, p. 149] whether the realcompactness of $X$ implies the realcompactness of $Y$ where $\varphi$ is a closed mapping from $X$ onto $Y$, or even whether the realcompactness of $\Phi^{-1}(Y) \mathrm{im}$ plies the realcompactness of $Y$. Concerning this problem, in Theorem 6.2, we shall discuss the structure of a space $Y$ which is the image of a realcompact space $X$ under an open $W Z$-mapping. From this theorem, we shall give a necessary and sufficient condition that $Y$ be realcompact. Moreover, from Theorem 6.2, we shall establish that if $\varphi$ is an open $W Z$-mapping from a realcompact space $X$ onto $Y$ such that the boundary $\mathscr{L} \varphi^{-1}(y)$ (or $\mathscr{L}_{X} \varphi^{-1}(y)$ ) of $\varphi^{-1}(y), y \in Y$, is compact, then $Y$ is also realcompact. This is a generalization of Frolík's theorem [2] (Theorem 6.5). As a further consequence of 6.2, the realcompactness is invariant under an open $W Z$-mapping if a space $X$ is any one of the following types; (1) $X$ is locally compact, (2) $X$ is weakly separable, (3) $X$ is connected, (4) $X$ is locally connected and (5) $X$ is perfectly normal. In Theorem 7.5, we shall prove, using Frolík's theorem [3], that if $X$ is locally compact, countably paracompact, normal space, then the image of $X$ under a closed mapping is realcompact when $X$ is realcompact. It seems to me that this is only one case for which realcompactness is proved to be invariant under a closed mapping without any additional condition. In the process of the proof of this theorem, we obtain that the image $Y$ of a locally compact, realcompact, normal space under a closed mapping $\varphi$ is locally compact if and only if $\mathscr{L}_{\varphi^{-1}}(y)$ is compact for every $y \in Y$.

1. Definitions and preliminaries, $\varphi: X \rightarrow Y$ is said to be a $Z$-mapping, according to Frolík [2], if $\varphi$ maps every zero set of $X$ to a closed set of $Y$. Moreover we shall define a $W Z$-mapping as a further generalization of a closed mapping. $\varphi$ is called a $W Z$-mapping if $\operatorname{cl}_{\beta X}\left(\varphi^{-1}(y)\right)=\Phi^{-1}(y)$ for every $y \in Y$. We shall say that a subset $F$ of $X$ has the property $\left({ }^{*}\right)$ if we have inf $\{f(x) ; x \in F\}>0$ for every $f \in C(X)$ which is positive on $F$. A subset $F$ of $X$ is said to be relatively pseudocompact if $f$ is bounded on $F$ for every $f \in C(X)$. A pseudocompact subset has the property $\left(^{*}\right)$ and a subset with the property $\left(^{*}\right)$ is always relatively pseudocompact, and hence every subset of a pseudocompact space is always relatively pseudocompact. We now list some properties with respect to these concepts. 
1.1. A closed mapping is always a Z-mapping.

1.2. A Z-mapping is always a WZ-mapping.

Proof. Let $z \in \Phi^{-1}(y)-\operatorname{cl}_{\beta X} \varphi^{-1}(y)$; then there is $f \in C(\beta X)$ such that $f(z)=0, f=1$ on $\operatorname{cl}_{\beta X} \varphi^{-1}(y)$ and $0 \leqq f \leqq 1$.

$$
M=X \cap\{x ; f(x) \leqq 1 / 2, x \in \beta X\}
$$

is a zero set of $X$. Since $\phi$ is a $Z$-mapping and $M \cap \varphi^{-1}(y)=\phi, \varphi(M)$ is closed and does not contain $y$. On the other hand, $f(z)=0$, and hence $z \in \operatorname{cl}_{\beta X} M$; this implies that

$$
y=\Phi(z) \in \Phi\left(\operatorname{cl}_{\beta X} M\right) \subset \operatorname{cl}_{\beta Y} \Phi(M)=\operatorname{cl}_{\beta Y} \varphi(M) .
$$

Since $\varphi(M)$ is closed in $Y, \operatorname{cl}_{\beta Y} \varphi(M) \cap Y=\varphi(M)$, and hence, $y \in Y$ implies $y \in \varphi(M)$. This is a contradiction.

1.3. Let $\varphi: X \rightarrow Y$ be a WZ-mapping. If either $X$ is normal or the boundary $\mathscr{L} \varphi^{-1}(y)$, for every $y \in Y$ is compact, then $\varphi$ is a closed mapping.

Proof. Let $F$ be a closed subset of $X$ and let $y \notin \varphi(F)$. It is easy to see, under the assumption of 1.3 , that there is $f \in C(X)$ such that $f=0$ on $\phi^{-1}(y), f=1$ on $F$ and $0 \leqq f \leqq 1$. Since $\phi$ is a $W Z$ mapping $\operatorname{cl}_{\beta X} \varphi^{-1}(y)=\Phi^{-1}(y)$ and $g=0$ on $\Phi^{-1}(y)$ where $g$ is the extension of $f$ over $\beta X$. $\Phi$ being closed, $Y-M$ is an open set containing $y$ and $\varphi(F) \subset M$ where $M=\Phi(\{z ; z \in \beta X, g(z) \geqq 1 / 2\})(y \notin M$ is obvious $)$. This means that $y \notin \overline{\varphi(F)}$, that is, $\varphi$ is closed.

1.4. Let $F$ be a closed relatively pseudocompact subset of $X$. If either $X$ is normal or $F$ is a zero set of $X$, then $F$ has the property (*) (see 3.3 below).

Proof. Let $f$ be a function of $C(X)$ and $f>0$ on $F$. Now suppose that $Z(f)=E \neq \phi$. If either $X$ is normal or $F=Z(g)$ for some $g \in C(X)$, then $E$ and $F$ are completely separated, i.e., there is a function $h \in C(X)$ such that $h=1$ on $E, h=0$ on $F$ and $0 \leqq h \leqq 1$. Then we have $Z(|f|+h)=\phi$ which implies $k=1 /(|f|+h) \in C(X)$. If $\inf \{f(x) ; x \in F\}=0$, then it is easy to see that $k$ is not bounded on the closed relatively pseudocompact subset $F$. This is a contradiction.

1.5. Every zero set of a pseudocompact space has the property (*) (by 1.4). 
1.6. Suppose that $\varphi$ is a mapping from $X$ onto $Y$ and every point of $Y$ is $G_{\delta}$. If a closed subset $F$ of $X$ has the property (*), then $\varphi(F)$ is closed.

Proof. Let $F$ be a closed subset of $X$ having the property $\left(^{*}\right)$ and let $y \notin \varphi(F)$. Since $y$ is a $G_{\delta}$-point, there is a function $f \in C(Y)$ with $f^{-1}(0)=\{y\}$ and $0 \leqq f \leqq 1 . h=f \varphi$ is positive on $F$, and hence $h>\alpha>0$ on $F$ because $F$ has the property $\left({ }^{*}\right)$. If $z \in \varphi(F)$, then there is a point $x \in F$ with $\varphi(x)=z$. Thus $f(z)=f(\varphi(x))=h(x)>\alpha$. This means that $\varphi(F) \subset f^{-1}[\alpha / 2,1]$, and hence $y \notin \overline{\varphi(F)}$, that is, $\varphi(F)$ is closed.

1.7. If, in 1.6, $X$ is pseudocompact, then $\varphi$ is always a Z-mapping (by 1.5 and 1.6).

The following theorems are known and useful in the sequel.

1.8. $X$ is realcompact if and only if for every point $x$ in $\beta X-X$ there is a function $f$ of $C(\beta X)$ such that $f>0$ on $X$ and $f(x)=0[4$, p. 119].

1.9. $X$ is pseudocompact if and only if any family $\left\{U_{n}\right\}$ of open sets of $X$, with $\overline{U_{n}} \cap \overline{U_{m}}=\phi(n \neq m)$, is not locally finite.

1.10. If $\left\{U_{n}\right\}$ is a locally finite family of open sets of a space $X$ with $\bar{U}_{n} \cap \bar{U}_{m}=\phi(n \neq m)$ and $\left\{a_{n}\right\}$ is a set of given positive real numbers and $\left\{x_{n}, x_{n} \in U_{n}\right\}$ is given, then there is a function $f$ of $C(X)$ such that $f=0$ on $X-\cup U_{n}, f\left(x_{n}\right)=a_{n}$, and $0 \leqq f \leqq a_{n}$ on $U_{n}$.

2. $Z$-mappings and pseudocompactness. A weakly separable space is a space with the first axiom of countability. The next conditions which are mutually equivalent, are known; (i) $X$ is compact (resp. countably compact), (ii) any mapping from $X$ onto $Y$ is closed for any space $Y$ (resp. any weakly separable space $Y$ ), and (iii) a projection $\varphi: Y \times X \rightarrow Y$ is closed for any space $Y$ (resp. any weakly separable space $Y)[5,8,12]$. In this section, we shall establish analogous theorems about pseudocompactness by means of $Z$-mappings.

Suppose that $X$ is not pseudocompact and let $\left\{W_{n}\right\}$ be a discrete family of open sets with $X-\cup W_{n}=S \neq \phi$. There are functions $f$ and $g$ of $C(X)$ by 1.10 such that (i) $f\left(x_{n}\right)=\varepsilon_{n},\left\{\varepsilon_{n}\right\} \downarrow 0$ and $f=0$ on $S$ where $x_{n}$ is a given point of $W_{n}$ and (ii) $g\left(x_{n}\right)=n, g=0$ on $S$ and $g(x)>0$ implies $f(x)>0$. Then $F=\{x ; g(x) \geqq 1 / 2\}$ is a zero set and

$$
\inf \{f(x) ; x \in F\}=0 \text {. }
$$


This shows that $F$ has not the property $\left(^{*}\right)$ and $f$ is not a $Z$-mapping from $X$ onto $f(X)$. Combining 1.5, 1.6 and the arguments above, we have the equivalences between (1), (2) and (3) in the following theorem.

THEOREM 2.1. The following conditions are equivalent for a space $X$.

(1) $X$ is pseudocompact.

(2) Every zero set of $X$ has the property (*).

(3) Any mapping from $X$ onto any space $Y$ such that every point of $Y$ is $G_{\delta}$, is always a Z-mapping.

(4) The projection $\varphi: Y \times X \rightarrow Y$ is a Z-mapping for any weakly separable space $Y$.

(5) The projection $\varphi: Y \times X \rightarrow Y$ is a Z-mapping for some nondiscrete weakly separable space $Y$.

Proof. (4) $\rightarrow(5)$ is obvious. We shall show $(1) \rightarrow(4)$. Suppose that there is a function $h \in C(X \times Y)$ such that $y \in \overline{\varphi(E)}-\varphi(E)$ where $E=h^{-1}(0)$. Let $\left\{W_{n}\right\}$ be a base of $y$ with

$$
\bar{W}_{n+1} \subset W_{n}(n=1,2, \cdots) \text {. }
$$

Since $\varphi^{-1}(y)=\{y\} \times X$ is pseudocompact and $h$ is positive on $\varphi^{-1}(y)$, there is a real number $\alpha>0$ such that $h \geqq \alpha$ on $\varphi^{-1}(y)$. For each $n$, we choose a point $y_{n}$ in $W_{n} \cap \varphi(E)$ (and hence $\left\{y_{n}\right\} \rightarrow y$ ) and a point $\left(y_{n}, x_{n}\right)$ in $E$. If $A=\left\{x_{n} ; n=1,2, \cdots\right\}$ has an accumulation point $x_{0}$, then $\left(y, x_{0}\right) \in E$, that is, $y=\varphi\left(y, x_{0}\right) \in \varphi(E)$. This is a contradiction. Thus $A$ must be a closed discrete subset of $X$. Let

$$
M=\{z ; h(z)<\alpha / 2\}
$$

and $F=\{z ; h(z) \leqq \alpha / 2)$. We choose an open set $U_{n}$, in $X$, containing $x_{n}$ and an open set $V_{n} \subset W_{n}$ in containing $y_{n} Y$ such that

$$
\bar{U}_{n}(\text { in } X) \cap \bar{U}_{m}(\text { in } X)=\phi(n \neq m), \bar{V}_{n} \times \bar{U}_{n} \subset M .
$$

$X$ being pseudocompact, there is an $x_{0}$ in $\bar{\cup}_{n_{i}}-\cup \bar{U}_{n_{i}}$ for some $\left\{n_{i}\right\}$. We have $\left(y, x_{0}\right) \in F$, i.e., $y=\varphi\left(y, x_{0}\right) \in \varphi(F)$. On the other hand, we have $\varphi^{-1}(y) \cap F=\phi$ since $F=\{z ; h(z) \leqq \alpha / 2\}$ and $h \geqq \alpha$ on $\varphi^{-1}(y)$. This is a contradiction.

$(5) \rightarrow(1)$ follows from the following theorem.

THEOREM 2.2. Suppose that $Y$ is a space in which there is a discrete subset $M=\left\{y_{n} ; y=1,2, \cdots\right\}$ which has an accumulation point $y_{0}$. If the projection $\varphi: Y \times X \rightarrow Y$ is a Z-mapping, then $X$ must be pseudocompact. 
Proof. We shall firstly show that there is a function $f \in C(Y)$ with $f\left(y_{n}\right)>0$ for every $y_{n} \in M$ and $f\left(y_{0}\right)=0$. Since $Y$ is completely regular, there is a function $f_{1} \in C(Y)$ with $f_{1}\left(y_{1}\right)=1, f_{1}=0$ on some neighborhood (briefly, nbd) $V_{1}$ of $y_{0}$ and $0 \leqq f_{1} \leqq 1$. Let $y_{i_{2}}$ be the point such that $y_{i_{2}} \in M \cap Z\left(f_{1}\right)$ and $i_{2}>m$ implies $f_{1}\left(y_{m}\right)>0$. Then there is a function $f_{2} \in C(Y)$ such that $f_{2}\left(y_{i_{2}}\right)=1, f_{2}=0$ on some nbd $V_{2}$ of $y_{0}, V_{2} \subset V_{1}$ and $0 \leqq f_{2} \leqq 1$ and $Z\left(f_{2}\right) \subset Z\left(f_{1}\right)$. Let $y_{i_{3}}$ be the point such that $y_{i_{3}} \in M \cap Z\left(f_{2}\right)$ and $i_{3}>m$ implies $f_{2}\left(y_{m}\right)>0$ and so on. Define $f(x)=\sum_{n=1}^{\infty}\left(1 / 2^{n}\right) f(x)$. Then $f(x)$ is continuous and $f\left(y_{0}\right)=0$ and $f>0$ on $M$.

If $X$ is not pseudocompact, there is a locally finite family $\left\{U_{n}\right\}$ of open sets with $U_{n} \cap U_{m}=\phi$ and there is a function $h \in C(X)$ such that $h \geqq 0$ on $X$ and $h\left(x_{n}\right)=1 / f\left(y_{n}\right)$ for some point $x_{n} \in U_{n}$ by 1.10. Define $H(y, x)=f(y) h(x) . \quad H(y, x)$ is continuous on $Y \times X$ and

$$
H\left(y_{0}, x\right)=0
$$

for every $x \in X$ and $H\left(y_{n}, x_{n}\right)=1$ for $n=1,2, \cdots$. Therefore we have $\left\{\left(y_{n}, x_{n}\right) ; n=1,2, \cdots\right\} \subset H^{-1}(1)$ and hence $M \subset \varphi\left(H^{-1}(1)\right)$. On the other hand, $y_{0} \notin \varphi\left(H^{-1}(1)\right)$. This shows that $\varphi$ is not a $Z$-mapping.

Even if $X$ is pseudocompact, a closed subset $F$ of $X$ with the property $\left({ }^{*}\right)$ is not necessarily pseudocompact. For instance, the space $D$ constructed in [4,5I, p. 79], which is a zero set of the pseudocompact space $\Psi$, is not pseudocompact.

Relating this example, we shall consider a countably compact space. If $X$ is not countably compact, then there are a discrete closed subset $A=\left\{x_{n} ; n=1,2, \cdots\right\}$ and a function $f \in C(X)$ such that

$$
f\left(x_{n}\right)=\varepsilon_{n},\left\{\varepsilon_{n}\right\} \downarrow 0 \text { and } f \geqq 0 \text { on } X .
$$

It is obvious that $A$ has not the property $\left({ }^{*}\right)$. Thus we see that $X$ is countably compact if and only if every closed subset of $X$ has the property $(*)$.

3. Mappings and the property (*). In this section we shall consider the relations between mappings given in $\S 1$ and the property $(*)$, and moreover give several examples. We shall say that $\varphi$ has the property $(*)$ if $\varphi^{-1}(y)$ has the property $(*)$ for every $y \in Y$.

3.1. (1) Let $\varphi: X \rightarrow Y$ be a mapping and every $\varphi^{-1}(y), y \in Y$, be relatively pseudocompact. If $\varphi$ is a Z-mapping, then $\varphi$ has the property $(*)$.

(2) If $\varphi: X \rightarrow Y$ is a WZ-mapping and $\varphi$ has the property (*), then $\varphi$ is a Z-mapping. 
Proof. (1). Suppose that there is a point $y$ in $Y$ such that $F=\varphi^{-1}(y)$ has not the property $\left({ }^{*}\right)$, that is, there exists a function $h \in C(X)$ which is positive on $F, h \geqq 0$ on $X$ and $h\left(x_{n}\right)=\varepsilon_{n},\left\{\varepsilon_{n}\right\} \downarrow 0$ for some sequence $\left\{x_{n}\right\}$ in $F$. We can find a family $\left\{W_{n}\right\}$ of open sets such that $\bar{W}_{n} \cap \bar{W}_{m}=\phi(n \neq m), \varepsilon_{n}-\rho_{n} \leqq h(x) \leqq \varepsilon_{n}+\rho_{n}$ on $W_{n}$ where $\min \left\{\varepsilon_{n}-\varepsilon_{n+1}, \varepsilon_{n-1}-\varepsilon_{n}\right\}=2 \rho_{n}$, and $x_{n} \in W_{n} . E=h^{-1}(0)$ is not empty because $E=\phi$ implies $1 / h \in C(X)$ and $1 / h$ is not bounded on a relatively pseudocompact subset $F$. We shall show that $\rho$ is not a $Z$-mapping. To do this, it is sufficient to show that $y \in \overline{\varphi(E)}$ because $E$ is a zero set and $y \notin \varphi(E)$. If $y \notin \overline{\varphi(E)}$, then there is a function $g \in C(Y)$ such that $g=1$ on $\overline{\varphi(E)}, g(y)=0$ and $0 \leqq g \leqq 1$. This implies that $g_{\varphi} \in C(X), g_{\varphi}=1$ on $E$ and $g_{\varphi}=0$ on $F$. The function $k=h+g_{\varphi}$ is positive, continuous on $X$, and hence $1 / k \in C(X)$. On the other hand, $1 / k$ is not bounded on $F$. This contradicts the fact that $F$ is relatively pseudocompact.

(2). Let $F=Z(f), f \in C^{*}(X)$ and $y \notin \varphi(F)$. Since $\varphi$ has the property $\left(^{*}\right)$, we have inf $\left\{f(x) ; x \in \varphi^{-1}(y)\right\}=\alpha>0$. Let $g$ be an extension of $f$ over $\beta X$; then $g \geqq \alpha$ on $\Phi^{-1}(y)=\operatorname{cl}_{\beta X} \varphi^{-1}(y)$.

$$
E=\{x ; x \in \beta X, g(x) \leqq \alpha / 2\}
$$

is compact and $y \notin \Phi(E)$. $\Phi(E)$ being compact, $V=\beta Y-\Phi(E)$ is an open subset (in $\beta Y$ ) containing $y$. Thus $V \cap Y$ is an open subset (in $Y$ ) containing $y$ and $\varphi(F) \cap(V \cap Y) \subset \Phi(E) \cap V \cap Y=\phi$. This implies that $y \overline{\oplus(F)}$, that is, $\varphi(F)$ is closed which shows that $\varphi$ is a $Z$-mapping.

From 3.1 we have

3.2. (1) If $\varphi$ is a Z-mapping from a pseudocompact space $X$ onto $Y$, then $\varphi$ has the property $\left(^{*}\right)$.

(2) If $\phi$ is a WZ-mapping from a countably compact space $X$ onto $Y$, then $\varphi$ is a Z-mapping.

We can not replace " $Z$-mapping" in (1) of 3.2 by " $W Z$-mapping" and " $Z$-mapping" in (2) of 3.2 by "closed mapping" respectively, as will be seen from examples 3.4 and 3.5 below respectively.

3.3. If $F$ is a $C^{*}$-embedded subset of $X$ with the property $\left({ }^{*}\right)$, then $F$ is pseudocompact. In particular, in a normal space, a closed subset with the property $\left({ }^{*}\right)$ is always countably compact (see 1.4).

Proof. If $F$ is not pseudocompact, then there is a function $f \in C(F)$ with $1 \geqq f>0$ and $\inf \{f(x) ; x \in F\}=0$. Let $g$ be an extension of $f$ over $X$; then $g>0$ on $F$ and inf $\{g(x) ; x \in F\}=0$ which is a contradiction. 
EXAMPLE 3.4. Let $X=W\left(\omega_{1}+1\right) \times W\left(\omega_{0}+1\right)-\left\{\left(\omega_{1}, \omega_{0}\right)\right\}$,

$$
Y=W\left(\omega_{1}+1\right)
$$

and let $\varphi: X \rightarrow Y$ be defined by $\varphi(y, x)=y$. Every $\varphi^{-1}(y), y \in Y$, is relatively pseudocompact. Since $\beta X=W\left(\omega_{1}+1\right) \times W\left(\omega_{0}+1\right)$, we have $\Phi^{-1}(y)=\operatorname{cl}_{\beta X} \varphi^{-1}(y)$, i.e., $\varphi$ is an open $W Z$-mapping. But $\varphi$ is not a $Z$-mapping by (1) of 3.2 because $\varphi^{-1}\left(\omega_{1}\right)$ has not the property $\left({ }^{*}\right)$ and $X$ is pseudocompact.

EXAMPLE 3.5. Let

$$
X=W\left(\omega_{1}+1\right) \times W\left(\omega_{1}+1\right)-\left\{\left(\omega_{1}, \omega_{1}\right)\right\}, Y=W\left(\omega_{1}+1\right)
$$

and let $\varphi: X \rightarrow Y$ be defined by $\varphi(y, x)=y$. Every $\varphi^{-1}(y)$ is compact except $y=\omega_{1}$ and $\varphi^{-1}\left(\omega_{1}\right)$ is countably compact. Thus $\varphi$ is an open $Z$-mapping by (2) of 3.2 . But $\phi$ is not closed because

$$
F=\left\{(y, x) ; x=\omega_{1}, y \in W\left(\omega_{1}\right)\right\}
$$

is closed but $\varphi(F)=W\left(\omega_{1}\right)$ is not closed in $Y$. (We notice that $X$ is countably compact.)

EXAMPLE 3.6. Let $X=W\left(\omega_{1}+1\right) \times W\left(\omega_{1}+1\right)-\left\{(y, x) ; y=\omega_{1}\right.$,

$$
\left.\omega_{0}<x \leqq \omega_{1}\right\}, Y=W\left(\omega_{1}+1\right)
$$

and let $\varphi: X \rightarrow Y$ be defined by $\varphi(y, x)=y$. Since

$$
Z=W\left(\omega_{1}\right) \times W\left(\omega_{1}+1\right)
$$

is pseudocompact and $\beta Z=Y \times Y, X$ is pseudocompact [9] and it is easy to see that every $\varphi^{-1}(y), y \in Y$, is compact. Thus $\varphi$ is an open compact mapping but not a $W Z$-mapping. $(\varphi: X \rightarrow Y$ is said to be compact if $\varphi^{-1}(y)$ is compact for every $y \in Y$.)

4. Extensions of open mappings. For an extension of an open mapping $\varphi: X \rightarrow Y$ where both spaces $X$ and $Y$ are normal, the following theorem is known: if either $\varphi$ is compact or $\varphi$ is closed, then $\Phi$ is open ([1], in which $\varphi$ is assumed to be a many-valued mapping). In this section, we shall show that if $\varphi$ is a (single-valued) $W Z$-mapping, then we can drop the assumption of normality of both spaces; that is, $\varphi$ is open if and only if $\Phi$ is open. Let $\varphi: X \rightarrow Y$ be a mapping. A function $f$ is said to be $\varphi$-bounded if $f$ is bounded on $\varphi^{-1}(y)$ for every $y \in Y$.

If $f \in C(X)$ is $\phi$-bounded, we put

$$
f^{i}(y)=\inf \left\{f(x) ; x \in \varphi^{-1}(y)\right\}, f^{s}(y)=\sup \left\{f(x) ; x \in \varphi^{-1}(y)\right\} ;
$$


these are real-valued functions defined on $Y$. The following lemma is useful.

Lemma 4.1. ([2]). Let $\varphi: X \rightarrow Y$ be a mapping and let $f \in C(X)$ be $\varphi$-bounded.

(i) If $\varphi$ is open, then $f^{s}\left(r e s p . f^{i}\right)$ is lower (resp. upper) semicontinuous.

(ii) If $\varphi$ is closed, then $f^{s}\left(r e s p . f^{i}\right)$ is upper (resp. lower) semicontinuous.

(iii) If $\varphi$ is a WZ-mapping, then $f^{s}$ (resp. $f^{i}$ ) is upper (resp. lower) semi-continuous.

Proof. (i) and (ii) are essentially proved in [2]. (iii) is obtained in the following way: let $g$ be the extension of $f$ over $\Phi^{-1}(Y)$; by (ii) $g^{s}$ (resp. $g^{i}$ ) is upper (resp. lower) semi-continuous on $Y$ because $\Phi$ is a closed mapping. Since $\phi$ is a $W Z$-mapping, we have

$$
g^{s}=f^{s} \text { and } g^{i}=f^{i} .
$$

This completes the proof.

If $\varphi$ is an open WZ-mapping, then $f^{s}$ and $f^{i}$ are continuous on $Y$ for every $\varphi$-bounded function $f \in C(X)$ by 4.1 .

As applications of 4.1 we have the following 4.2 and 4.3 .

4.2. If $\varphi$ is an open WZ-mapping from $X$ onto a pseudocompact space $Y$ such that $\varphi^{-1}(y)$ is relatively pseudocompact for every $y \in Y$, then $X$ is pseudocompact.

This is a generalization of a theorem of Hanai and Okuyama [6] and our proof is simpler than theirs; that is, 4.2 follows from the facts that for any $f \in C(X), f$ is $\varphi$-bounded, and hence $f^{s}$ (resp. $f^{i}$ ) is bounded by (iii) and continuous on $Y$ by the note above which concludes that $f$ is bounded on $X$.

4.3. If $\varphi$ is a WZ-mapping from $X$ onto a countably compact space $Y$ such that $\varphi^{-1}(y)$ is relatively pseudocompact for every $y \in Y$, then $X$ is pseudocompact.

Proof. Let $f$ be any function of $C(X)$; then $|f|$ is $\varphi$-bounded and $|f|^{\text {s }}$ is upper semi-continuous by (iii). Since a space is countably compact if and only if every upper semi-continuous function is bounded above [10], we see that $|f|^{s}$ must be bounded above, that is, $f$ is bounded. This means that $X$ is pseudocompact. 
TheOREM 4.4. (i) A mapping $\varphi: X \rightarrow Y$ is a WZ-mapping if and only if $\varphi(U \cap X)=\Phi(U) \cap Y$ for every open set $U$ of $\beta X$.

(ii) If $\varphi: X \rightarrow Y$ is a WZ-mapping, then $\varphi$ is open if and only if $\Phi$ is open.

Proof. (i). Necessity. It is sufficient to prove that $y \in \Phi(U) \cap Y$ implies $y \in \varphi(U \cap X)$. This follows from the fact that

$$
\varphi^{-1}(y) \cap(U \cap X) \neq \phi
$$

if and only if $\Phi^{-1}(y) \cap U \neq \phi$ for every open set $U$ of $\beta X$ since $\varphi$ is a $W Z$-mapping.

Sufficiency. If $x \in \Phi^{-1}(y)-\operatorname{cl}_{\beta X} \varphi^{-1}(y)$, then there is an open set $U$ (in $\beta X$ ) containing $x$ which is disjoint from $\mathrm{cl}_{\beta X} \varphi^{-1}(y)$. This means that $y \notin \varphi(U \cap X)$, which contradicts $y \in \Phi(U)$.

(ii). It is sufficient, by (i), to show that the openness of $\varphi$ implies the openness of $\Phi$. Let $x^{*}$ be any point in $\beta X$ and let $U$ be an open set of $\beta X$ containing $x^{*}$. There exists a function $f \in C(\beta X)$ such that $0 \leqq f \leqq 1, f\left(x^{*}\right)=1, f=0$ on $\beta X-U$ and $\operatorname{cl}_{\beta X} V \subset U$ where

$$
V=\{x ; f(x)>0\} \text {. }
$$

We have, by 4.1, $(f \mid X)^{s} \in C(Y)$. Let us denote by $g$ the extension of $(f \mid X)^{s}$ over $\beta Y$. Then $g\left(\Phi\left(x^{*}\right)\right)=1$ and $W=\{y ; g(y)>1 / 2\}$ is open in $\beta Y$. We shall prove that $W \subset \Phi\left(\operatorname{cl}_{\beta X} V\right)$. Suppose that there is a point $z$ in $W$ such that $\Phi^{-1}(z) \cap \Phi^{-1} \Phi\left(\operatorname{cl}_{\beta X} V\right)=\phi$. Then $f=0$ on $\Phi^{-1}(S)$ where $S$ is an open subset, contained in $W$, containing $z$ with $S \cap \Phi\left(\operatorname{cl}_{\beta X} V\right)=\phi$. This implies that $g \mid Y=0$ on $S$ which is impossible. This theorem will be used in $\S 6$.

5. Inverse images of realcompact spaces. Let $\alpha$ be a collection of coverings of $X$. A centred family $\mathscr{C l}$ of subsets of $X$ (i.e., with the finite intersection property) is said to be $\alpha$-Cauchy if for every $\mathfrak{A} \in \alpha$, there exist $A \in \mathfrak{A}$ and $M \in \mathbb{C}$ with $M \subset A$. We shall say that $\alpha$ is complete if

$$
\cap-\overline{l l} \neq \phi
$$

for every $\alpha$-Cauchy $\mathscr{C}$, according to Frolík [3]. In the sequel, we consider only countable coverings consisting of cozero-sets where a set is said to be a cozero-set if it is the complement of a zero set. We denote by $\alpha_{c}$ the collection of all such coverings and moreover by $\alpha_{p c}\left(\right.$ resp. $\alpha_{1 c}$ and $\alpha_{s c}$ ) the subcollection of $\alpha_{c}$ with the point-finite property (resp. with the locally finite property and with the star-finite property). If $\alpha$ is a collection of countable coverings of $X$, then define $\overline{\mathfrak{A}^{\beta}}=\bigcup\left\{\mathrm{cl}_{\beta X} A ; A \in \mathfrak{A}\right\}$ for every $\mathfrak{A} \in \alpha . \overline{\mathfrak{A}}^{\beta}$ is $\sigma$-compact and hence 
$Z=\cap\left\{\overline{\mathfrak{A}}^{\beta} ; \mathfrak{A} \in \alpha\right\}$ is realcompact and $X \subset \sim X \subset Z \subset \beta X$ where $v X$ denotes the Hewitt's realcompactification of $X$.

Lemma 5.1. Let $\mathscr{C l}$ be a centred maximal family of zero sets. Then $\mathscr{C l}$ is $\alpha$-Cauchy if and only if $\mathscr{C l}$ has the countable intersection property where $\alpha$ is any one of $\alpha_{c}, \alpha_{p c}, \alpha_{1 c}$ and $\alpha_{s c}$.

Proof. Necessity. Suppose that there is $\left\{Z_{n}\right\}$ in $\mathscr{C l}$ with

$$
\cap Z_{n}=\phi
$$

where $Z_{n}=Z\left(f_{n}\right), 0 \leqq f_{n} \leqq 1$ and $f_{n} \in C(X)$. Then $f=\Sigma\left(f_{n} / 2^{n}\right)$ is a positive continuous function on $X$.

$$
A_{n}=\{x ; 1 /(n+2)<f(x)<1 / n\}
$$

is a cozero-set because $A_{n}=X-Z\left(g_{n}\right)$ where $g_{n}=(-|f-a|+a) \vee 0$ and $a=(1 /(n+2)+1 / n) / 2$. It is easy to see that $\mathfrak{A}=\left\{A_{n}\right\} \in \alpha_{s c}$. If there is $Z \in \mathscr{C l}$ with $Z \subset A_{n}$ for some $n$, then

$$
B=Z \cap Z_{1} \cap \cdots \cap Z_{n+2} \neq \phi
$$

and we have $1 /(n+2)<f<1 / n$ on $B$. On the other hand,

$$
f<1 /(n+2)
$$

on $B$ by the method of construction of $f$. Thus $/ /$ is not $\alpha_{s c^{-}}$ Cauchy.

Sufficiency. It is sufficient to show that if $\mathfrak{M}$ is not $\alpha_{c}$-Cauchy, then $/ l$ has not the countable intersection property. Since $/ l$ is not $\alpha_{c}$-Cauchy, there exists

$$
\mathfrak{U}=\left\{A_{n} ; A_{n}=Z_{n}^{c}, Z_{n}=Z\left(f_{n}\right), f_{n} \in C(X)\right\} \in \alpha_{c}
$$

such that $M \not \subset A_{n}$ for every $n$ and every $M \in \mathscr{C l}$. Hence $M \cap Z_{n} \neq \phi$ for every $M \in \mathscr{C}$. $\mathscr{C l}$ being maximal, $Z_{n} \in \mathscr{l}$. Since $\left\{Z_{n}^{c}\right\}$ is a covering of $X$, we have $\cap Z_{n}=\dot{\rho}$, and hence $\mathscr{C l}$ has not the countable intersection property.

LEMMA 5.2. The following statements are equivalent.

(1) $X$ is realcompact.

(2) A centred maximal family of zero sets with the countable intersection property has the total nonempty intersection.

(3) $\alpha$ is complete where $\alpha$ is any one of $\alpha_{c}, \alpha_{p c}, \alpha_{1 c}$ and $\alpha_{s c}$.

Proof. (1) $\leftrightarrow(2)$ is already proved in [4].

$(3) \rightarrow(1)$. If $p \in \cup X-X$, then $\mathscr{C}=\left\{Z ; p \in \operatorname{cl}_{\beta X} Z, Z\right.$ is a zero set of $X\}$ is a maximal centred family with the countable intersection 
property, and hence by 5.1, $\mathscr{C}$ is $\alpha_{c}$-Cauchy. Since $\alpha_{c}$ is complete, $\cap \mathscr{N} \neq \phi$ and it is obvious that $\cap\left\{\mathrm{cl}_{\beta x} Z: Z \in \mathscr{C}\right\}=\{p\}$. This is a contradiction, that is, $\cup X=X$.

$(1) \rightarrow(3)$. It is sufficient to prove that the realcompactness implies the completeness of $\alpha_{s c}$. Let $\alpha_{N}$ be the family of all countable normal open coverings; then $\alpha_{N}$ is complete since $X$ is realcompact. On the other hand, $\alpha_{s c}$-Cauchy family is $\alpha_{N}$-Cauchy family. Therefore we see $\alpha_{s c}$ is complete.

TheOREM 5.3. Let $\varphi: X \rightarrow Y$ be a Z-mapping and let every $\varphi^{-1}(y), y \in Y$, be a $C^{*}$-embedded realcompact subset of $X$. If $Y$ is realcompact, then so is also $X$.

Proof. Let $\mathscr{C}$ be a maximal centred $\alpha_{c}$-Cauchy family consisting of zero sets of $X$; then $\mathscr{C}$ has the countable intersection property by 5.1. Thus by 5.2 it is sufficient to show that $\mathscr{C}$ has the total nonempty intersection. Since $\varphi(\mathscr{M})$ is $\alpha_{c}$-Cauchy (in $Y$ ) and $Y$ is realcompact, we have $y \in \cap \varphi(\mathscr{C})$ for some point $y$ by 5.2. $\varphi$ being a $Z$-mapping, $\varphi(M)=\overline{\varphi(M)}$ for every $M \in \mathscr{L}$. Since $M, N \in \mathscr{C l}$ implies $M \cap N \in \mathscr{C}, \mathscr{L} \cap \varphi^{-1}(y)$ has the finite intersection property on $\varphi^{-1}(y)$. Let $\mathfrak{A}=\left\{\varphi^{-1}(y)-Z\left(g_{n}\right) ; n=1,2, \cdots\right\}$ be a covering of $\varphi^{-1}(y)$ where $g_{n} \in C\left(\varphi^{-1}(y)\right)$ and $g_{n}$ is bounded. Without loss of generality we can assume that $0 \leqq g_{n} \leqq 1$ for each $n$. Let $f_{n}$ be an extension of $g_{n}$ over $X$ and define $f=\sum\left(f_{n} / 2^{n}\right) . \quad f$ is continuous and $Z(f) \cap \varphi^{-1}(y)=\phi . \quad Y$ being completely regular and $\varphi$ being a $Z$-mapping, there is $h \in C(Y)$ with $0 \leqq h \leqq 1, h(\varphi Z(f))=1$ and $h(y)=0$.

$$
\left\{X-Z(h \varphi), X-Z\left(f_{n}\right) ; n=1,2, \cdots\right\}
$$

is a covering of $X$. We shall show that $M \not \subset X-Z(h \varphi)$ for every $M \in \mathscr{M}$. Suppose that there is a set $M \in \mathscr{M}$ such that

$$
M \subset X-Z(h \varphi) \text {. }
$$

Since $\varphi^{-1}(y) \subset Z(h \varphi)$, we have $M \cap \varphi^{-1}(y)=\phi$, but this contradicts the fact that $M \cap \varphi^{-1}(y) \neq \phi$ for every $M \in \mathscr{M}$. Thus there are $M \in \mathscr{M}$ and $n$ with $M \subset X-Z\left(f_{n}\right)$, that is, $\mathscr{M} \cap \varphi^{-1}(y)$ is $\alpha_{c}$-Cauchy (on $\left.\varphi^{-1}(y)\right)$. Since $\varphi^{-1}(y)$ is realcompact, we have $\cap\left(\mathscr{C} \cap \varphi^{-1}(y)\right) \neq \phi$. This means $\cap \mathscr{C} \neq \phi$. Therefore $X$ is realcompact.

THEOREM 5.4. If $\varphi$ is a closed mapping from a normal space $X$ to a realcompact space $Y$ such that every $\varphi^{-1}(y), y \in Y$, is realcompact, then $X$ is also realcompact.

6. Open WZ-mappings and realcompactness. A point $p$ is said 
to be a $P$-point of $X$ if every continuous function defined on $X$ is constant on some nbd of $p$. A space $X$ is called a $P$-space if every point of $X$ is a $P$-point of $X$.

In the following, let $\varphi: X \rightarrow Y$ be an open $W Z$-mapping, and we divide both spaces $X$ and $Y$ into classes in the following way: $X_{d}=$ $\left\{x ; \varphi(x)\right.$ is isolated and $\varphi^{-1} \varphi(x)$ is not compact $\}, X_{c d}=\{x ; \varphi(x)$ is isolated and $\varphi^{-1} \varphi(x)$ is compact $\}, X_{e}=\left\{x ; x \notin X_{d} \cup X_{c d}\right.$ and $\varphi^{-1} \varphi(x)$ is not compact\},

$$
\begin{aligned}
& X_{c e}=X-X_{d}-X_{c d}-X_{e}, Y_{d}=\varphi\left(X_{d}\right), \\
& Y_{c d}=\varphi\left(X_{c d}\right), Y_{e}=\varphi\left(X_{e}\right) \text { and } Y_{c e}=\varphi\left(X_{c e}\right) .
\end{aligned}
$$

Lemma 6.1. If $\varphi: X \rightarrow Y$ is an open WZ-mapping, $y^{*} \in Y_{e}$ and if there is a function $f \in C(\beta X)$ such that $0 \leqq f \leqq 1, f>0$ on $X$ and $f\left(x^{*}\right)=0$ for some $x^{*} \in \Phi^{-1}\left(y^{*}\right)-\varphi^{-1}\left(y^{*}\right)$, then $Z_{\beta X}\left(f^{i} \Phi\right)$ is a neighborhood (in $\beta X)$ of $\Phi^{-1}\left(y^{*}\right)$, equivalently, $Z_{\beta Y}\left(f^{i}\right)$ is a neighborhood (in $\beta Y$ ) of $y^{*}$. (We notice that $\Phi$ is open by 4.4)

Proof. Suppose that $Z_{\beta Y}\left(f^{i}\right)$ is not a nbd of $y^{*}$, i.e., $Z_{Y}\left(f^{i}\right)$ is not a nbd of $y^{*}$. Let us put $h=f^{i} \mid Y, \alpha_{2 n}=1 / 2 n-1 /(2 n+1)$ and

$$
\begin{aligned}
a_{n} & =1 / 2 n-(4 / 7) \cdot \alpha_{2 n}, \\
b_{n} & =1 / 2 n+(4 / 7) \cdot \alpha_{2 n-1} \\
c_{n} & =1 /(2 n+1)-(4 / 7) \cdot \alpha_{2 n+1}, \\
d_{n} & =1 /(2 n+1)+(4 / 7) \cdot \alpha_{2 n} \\
F_{n} & =\varphi^{-1} h^{-1}\left[\alpha_{n}, b_{n}\right], \\
E_{n} & =\varphi^{-1} h^{-1}\left[c_{n}, d_{n}\right] .
\end{aligned}
$$

It is easy to see that either $\operatorname{cl}_{\beta X}\left(\cup F_{n}\right)$ or $\operatorname{cl}_{\beta X}\left(\cup E_{n}\right)$ contains $x^{*}$, say $\operatorname{cl}_{\beta X}\left(\cup F_{n}\right) \ni x^{*}$. Let us put $q_{n}=\left(f_{n}-b_{n}\right) \vee 0$ and

$$
k_{n}=\left|h_{\varphi}-\beta_{n}\right| \vee\left\{b_{n}-\beta_{n}\right\}-\left\{b_{n}-\beta_{n}\right\}
$$

where $\beta_{n}=\left(a_{n}+b_{n}\right) / 2$; then $q_{n} \in C(\beta X), k_{n} \in C(X), A_{n}=\{x ; x \in \beta X$, $\left.f(x) \leqq b_{n}\right\}=Z_{\beta X}\left(q_{n}\right), F_{n}=Z_{X}\left(k_{n}\right)$ and $\left\{G_{n} ; n=1,2, \cdots\right\}$ is locally finite family of zero sets of $X$ where $G_{n}=Z_{X}\left(q_{n}+k_{n}\right)=F_{n} \cap A_{n}$. We can assume that every $G_{n}$ is not empty.

Next we shall prove that $\cup G_{n}$ is a zero set. If we put

$$
t_{n}=1 / 2 n-(5 / 7) \cdot \alpha_{2 n}, s_{n}=1 / 2 n+(5 / 7) \cdot \alpha_{2 n-1}
$$

and $B_{n}=\left\{x ; x \in \beta X, f(x)<s_{n}\right\}$, then $U_{n}=\varphi^{-1} h^{-1}\left(t_{n}, s_{n}\right)$ is an open set containing $F_{n}$ and $W_{n}=U_{n} \cap B_{n}$ is also an open set such that $G_{n} \subset W_{n}$ and $\bar{W}_{n} \subset \varphi^{-1} h^{-1}\left[t_{n}, s_{n}\right]$. Since $\bar{W}_{n} \cap \bar{W}_{m}=\phi$ and $x \in \bar{\cup} \bar{W}_{n}-\cup \bar{W}_{n}$ implies $f(x)=0,\left\{W_{n}\right\}$ is a discrete collection of open sets of $X$ be- 
cause $f>0$ on $X$. If $x \notin B_{n}$, then $f(x) \geqq s_{n}, k_{n}(x) \geqq 0$, and hence

$$
k_{n}(x)+q_{n}(x) \geqq q_{n}(x)>s_{n}-b_{n} a_{n}-t_{n}=p_{n}>0 \text {. }
$$

If $x \notin U_{n}$, then $\left|h \varphi(x)-\beta_{n}\right|>\beta_{n}-t_{n}, q_{n}(x) \geqq 0$, and hence

$$
k_{n}(x)+q_{n}(x) \geqq k_{n}(x)>\beta_{n}-t_{n}-b_{n}+\beta_{n}=a_{n}-t_{n}=p_{n}>0 .
$$

Let us put $g_{n}(x)=\left\{\left(k_{n}(x)+q_{n}(x)\right) \wedge p_{n}\right\} \times\left(1 / p_{n}\right)$. Then

$$
g_{n}=1 \text { on } X-W_{n} \text { and } x \in G_{n}
$$

if and only if $g_{n}(x)=0$. Define

$$
g(x)= \begin{cases}1 & \text { for } x \in X-\cup W_{n} \\ g_{n}(x) & \text { for } x \in W_{n}-G_{n} \\ 0 & \text { for } x \in \cup G_{n} .\end{cases}
$$

Since $\left\{W_{n}\right\}$ is a discrete collection, $g(x)$ is continuous and $Z(g)=\cup G_{n}$, that is, $\cup G_{n}$ is a zero set.

Since $Z(g) \cap Z(h \varphi)=\phi$, we have $\operatorname{cl}_{\beta X} Z(g) \cap \mathrm{cl}_{\beta X} Z(h \varphi)=\phi$, and hence $y^{*} \notin \Phi(Z(g))$ because $\mathrm{cl}_{\beta X} Z(h \varphi) \supset \Phi^{-1}\left(y^{*}\right)$ (notice; $\varphi$ is a $W Z$-mapping).

Replacing $a_{n}, b_{n}, t_{n}$ and $s_{n}$ by $a_{n}^{\prime}=1 / 2 n-(5 / 7) \cdot \alpha_{2 n}, b_{n}^{\prime}=1 / 2 n+$ $(5 / 7) \cdot \alpha_{2 n-1}, t_{n}^{\prime}=1 / 2 n-(6 / 7) \cdot \alpha_{2 n}$ and $s_{n}^{\prime}=1 / 2 n+(6 / 7) \cdot \alpha_{2 n-1} \quad$ respectively, we can define and construct $F_{n}^{\prime}, q_{n}^{\prime}, \beta_{n}^{\prime}, k_{n}^{\prime}, A_{n}^{\prime}, G_{n}^{\prime}, p_{n}^{\prime}, g_{n}^{\prime}$ and $g^{\prime}$ using methods similar to definitions and constructions of $F_{n}, q_{n}, \beta_{n}, k_{n}$, $A_{n}, G_{n}, p_{n}, g_{n}$ and $g$ respectively in the arguments above. Then

$$
G_{n} \subset G_{n}^{\prime}, Z(g) \subset Z\left(g^{\prime}\right), Z\left(g^{\prime}\right) \cap Z(h \varphi)=\phi
$$

and $y^{*} \notin \Phi\left(Z\left(g^{\prime}\right)\right)$. Thus there exists a nbd $W$ (in $Y$ ) of $y^{*}$ with

$$
W \cap \Phi\left(Z\left(g^{\prime}\right)\right)=\dot{\phi} \text {. }
$$

On the other hand, $x^{*} \in \operatorname{cl}_{\beta X}\left(\cup F_{n}\right)$ and $y^{*} \in Y$ implies $\left.y^{*} \in \overline{\cup \varphi(F}_{n}\right)$, and hence there is a point $y$ in $\varphi\left(F_{m}\right) \cap W$ for some $m$, that is

$$
a_{m} \leqq h(y) \leqq b_{m} \text {. }
$$

This shows that there exists a point $x$ of $\varphi^{-1}(y)$ with $x \in A_{m}^{\prime}$ and $x \in F_{m}^{\prime}$. Since $G_{m}^{\prime}=A_{m}^{\prime} \cap F_{m}^{\prime}, y \in \varphi\left(G_{m}^{\prime}\right)$. This contradicts $W \cap \Phi\left(Z\left(g^{\prime}\right)\right)=\phi$.

The following theorem indicates the structure of the image of a realcompact space under an open $W Z$-mapping.

THEOREM 6.2. Let $\varphi$ be an open WZ-mapping from a realcompact space $X$ onto $Y$.

(i) Every point $y \in Y_{e}$ is a nonisolated $P$-point of $Y$, and hence $Y_{e} \cup Y_{d}$ is an open $P$-subspace of $Y$ and $Y_{c e} \cup Y_{c d}$ is closed in $Y$. 
(We shall prove in 6.5 that $Y_{e}=\phi$ implies the realcompactness of $Y$ ).

(ii) If $Y$ is not realcompact, then every point $y^{*}$ of $\cup Y=Y$ is a P-point of $\beta Y$ and $Y_{c e} \cup Y_{c d}$ is closed in $\cup Y$.

Proof. (i). Let $y \in Y_{e}$ and $h \in C(\beta Y)$ with $h(y)=0$ and let

$$
x^{*} \in \Phi^{-1}(y)-\phi^{-1}(y) \text {. }
$$

$X$ being realcompact, there is a function $f \in C(\beta X)$ such that

$$
0 \leqq f \leqq 1, f\left(x^{*}\right)=0
$$

and $f>0$ on $X . \quad k=f+h \Phi$ is continuous and $k>0$ on $X$ and

$$
k\left(x^{*}\right)=0 .
$$

By 6.1, $Z\left(k^{i}\right)$ is a nbd (in $\beta Y$ ) of $y$. On the other hand $k^{i} \geqq h$ implies $Z\left(k^{i}\right) \subset Z(h)$. This shows that $h$ vanishes on some nbd of $y$, i.e., $y$ is a $P$-point of $Y$. Thus $Y_{e} \cup Y_{d}$ becomes to be a $P$-space. Since $k^{i}(y)>0$ for every $y \in Y_{c e} \cup Y_{c d}, Y_{e} \cup Y_{d}$ is open in $Y$ and hence $Y_{c e} \cup Y_{c d}$ is closed in $Y$.

(ii). Let $y^{*} \in \cup Y-Y, x^{*} \in \Phi^{-1}\left(y^{*}\right)$ and let $f$ be a function of $C(\beta X)$ with $0 \leqq f \leqq 1, f\left(x^{*}\right)=0, f>0$ on $X$. Let us put $X_{0}=\Phi^{-1}(Y)$. If $Z_{\beta X}(f) \cap X_{0}=\phi$, then $Z_{\beta Y}\left(f^{i}\right) \cap Y=\phi$ since every $\Phi^{-1}(y), y \in Y$, is compact and $f>0$ on $X_{0}$, and hence $f^{i}>0$ on $Y$ and $f^{i}\left(y^{*}\right)=0$. Thus we have $1 / f^{i} \in C(Y)$ and $1 / f^{i}$ can not be continuously extended over $y^{*}$. But this is impossible since $y^{*} \in v Y-Y$. Thus we have $Z_{\beta X}(f) \cap X_{0} \neq \phi$ which implies $Z_{Y}\left(f^{i}\right) \neq \phi$. For every $y \in Y_{c e} \cup Y_{c d}$, $f>\alpha(y)$ on $\varphi^{-1}(y)$ because $\varphi^{-1}(y)$ is compact where $\alpha(y)$ is some real number. $Z_{\beta Y}\left(f^{i}\right) \cap Y$ is an open-closed subset of $Y\left(\subset Y_{e} \cup Y_{d}\right)$ by (i) and $\operatorname{cl}_{\beta X}\left(Z\left(f^{i}\right) \cap Y\right)\left(\subset \operatorname{cl}_{\beta Y} Z\left(f^{i}\right)=Z\left(f^{i}\right)\right)$ is also open-closed in $\beta Y$. This shows that $y^{*} \in \cup\left(Z\left(f^{i}\right) \cap Y\right)$ because

$$
v Y=v\left(Z\left(f^{i}\right) \cap Y\right) \cup v\left(Y-Z\left(f^{i}\right)\right)
$$

and $\quad v\left(Z\left(f^{i}\right) \cap Y\right) \cap v\left(Y-Z\left(f^{i}\right)\right)=\phi \quad$ (we notice $\left.Z\left(f^{i}\right)=Z_{\beta Y}\left(f^{i}\right)\right)$. Since $Z\left(f^{i}\right) \cap Y$ is a $P$-space, so is also $v\left(Z\left(f^{i}\right) \cap Y\right)$ and every point of $v\left(Z\left(f^{i}\right) \cap Y\right)$ is a $P$-point of $v\left(Z\left(f^{i}\right) \cap Y\right)$ and hence of $\beta Y$ $[4, p .211]$.

From the argument above, every point $y^{*} \in v Y-Y$ has a nbd which is disjoint from $Y_{c e} \cup Y_{c d}$, and by (i) every point of $Y_{e} \cup Y_{d}$ has also a nbd which is disjoint from $Y_{c e} \cup Y_{c d}$. Thus $Y_{c e} \cup Y_{c d}$ is closed in $\cup Y$.

If $\beta Y-Y$ contains a $P$-point $p$ of $\beta Y$, then it is known that every function $f \in C(Y)$ can be continuously extended over $p$, and hence, $Y$ is not realcompact. The converse is not necessarily true. 
Such an example is given by the space in Example 3.4, that is, $Y=W\left(\omega_{1}+1\right) \times W\left(\omega_{0}+1\right)-\left\{\left(\omega_{1}, \omega_{0}\right)\right\}$ is not realcompact but $\beta Y-Y$ consists of only one point $\left(\omega_{1}, \omega_{0}\right)$ which is not a $P$-point of $\beta Y$.

But if $Y$ is the image of a realcompact space $X$ under an open $W Z$-mapping, then Theorem 6.2 concludes the following: the fact that $Y$ is not realcompact implies that $\beta Y-Y$ contains a $P$-point of $\beta Y$. Thus the equivalence of (1) and (2) in the following Theorem 6.3 is obtained.

Let $y^{*} \in \beta Y-Y$. We denote by $0\left(y^{*}\right)$ the set of all functions of $C(X)$ such that $\operatorname{cl}_{\beta X} Z_{X}(f)$ is a nbd of $\Phi^{-1}\left(y^{*}\right)$, and

$$
Z\left(0\left(y^{*}\right)\right)=\left\{Z_{x}(f) ; f \in 0\left(y^{*}\right)\right\} .
$$

$0\left(y^{*}\right)$ is a $Z$-ideal of $C(X)$.

THEOREM 6.3. Let $\varphi$ be an open WZ-mapping from a realcompact space $X$ onto $Y$; then the following statements are equivalent.

(1) $Y$ is realcompact.

(2) There is no P-point of $\beta Y$ in $\beta Y-Y$.

(3) $Z\left(0\left(y^{*}\right)\right)$ is not closed under countable intersection for every $y^{*} \in \beta Y-Y$.

(4) There is a function $g \in C(\beta X)$ such that $\Phi^{-1}\left(y^{*}\right) \subset Z_{\beta x}(g)$ but $Z_{\beta X}(g)$ is not a nbd of $\Phi^{-1}\left(y^{*}\right)$ for every $y^{*} \in \beta Y-Y$.

Proof. (2) $\rightarrow(3)$. Suppose that there is a point $y^{*}$ such that $Z\left(0\left(y^{*}\right)\right)$ is closed under countable intersection. Let $g$ be any function of $C(\beta Y)$ with $0 \leqq g \leqq 1$ and $g\left(y^{*}\right)=0$; then it is sufficient to show that $Z_{\beta Y}(g)$ is a nbd of $y^{*}$, i.e., $y^{*}$ is a $P$-point of $\beta Y$. Put $g=\left(g_{n} \vee 1 / n\right)-1 / n$ and $f_{n}=g_{n} \mid Y$. It is obvious that $\operatorname{cl}_{\beta Y} Z_{Y}\left(f_{n}\right)$ is a nbd of $y^{*}, f_{n} \varphi \in C(X)$ and $\varphi^{-1} Z_{Y}\left(f_{n}\right)=Z_{X}\left(f_{n} \varphi\right)$. If $\operatorname{cl}_{\beta X} Z_{X}\left(f_{n}\right)$ is not a nbd of $\Phi^{-1}\left(y^{*}\right)$, then $Z_{X}\left(f_{n} \varphi\right)$ does not contain $X \cap U$ for any nbd $U$ of $\Phi^{-1}\left(y^{*}\right)$. Since $\varphi$ is open and $\varphi\left(Z_{X}\left(f_{n} \varphi\right)\right)=\varphi \varphi^{-1} Z_{Y}\left(f_{n}\right)=Z_{y}\left(f_{n}\right), \varphi(X \cap U)$ is open and $\varphi(X \cap U)$ is not contained in $Z_{Y}\left(f_{n}\right)$. This contradicts the fact that $\operatorname{cl}_{\beta Y} Z_{Y}\left(f_{n}\right)$ is a nbd of $y^{*}$. Therefore $\operatorname{cl}_{\beta X} Z_{X}\left(f_{n} \varphi\right)$ is a nbd of $\Phi^{-1}\left(y^{*}\right)$. Since $Z_{x}\left(f_{n} \varphi\right) \in Z\left(0\left(y^{*}\right)\right)$ and $Z\left(0\left(y^{*}\right)\right)$ is closed under countable intersection, there is a function $k \in 0\left(y^{*}\right)$ with $\cap Z_{X}\left(f_{n} \varphi\right)=Z_{X}(k)$. Since $k \in 0\left(y^{*}\right)$, $\operatorname{cl}_{\beta X} Z(k)$ is a nbd of $\Phi^{-1}\left(y^{*}\right)$ and $\Phi\left(\operatorname{cl}_{\beta X} Z_{X}(k)\right)$ is a nbd of $y^{*}$ because $\Phi$ is open by 4.4. On the other hand, $x \in Z_{X}(k)$ implies $\left(f_{n} \varphi\right)(x)=0$ for every $n$, and hence we have $\varphi(x) \in Z_{Y}(g \mid Y)$, i.e., $\varphi\left(Z_{X}(k)\right) \subset Z_{Y}(g \mid Y)$. We have

$$
\Phi\left(\operatorname{cl}_{\beta X} Z_{X}(k)\right) \subset \operatorname{cl}_{\beta Y} \Phi\left(Z_{X}(k)\right)=\operatorname{cl}_{\beta Y}\left(\varphi Z_{X}(k)\right) \subset \operatorname{cl}_{\beta Y} Z_{Y}(g \mid Y) \subset Z_{\beta Y}(g) .
$$

This shows that $Z_{\beta Y}(g)$ is a nbd of $y^{*}$.

$(3) \rightarrow(4)$. Since $Z\left(0\left(y^{*}\right)\right)$ is not closed under countable intersec- 
tion, there is a function $f_{n} \in 0\left(y^{*}\right)(n=1,2, \cdots)$ and $\operatorname{cl}_{\beta X}\left(\cap Z_{X}\left(f_{n}\right)\right)$ is not a nbd of $\Phi^{-1}\left(y^{*}\right)$. Let $f=\sum\left(1 / 2^{n}\right)\left(\left|f_{n}\right| /\left(1+\left|f_{n}\right|\right)\right.$. If

$$
z^{*} \in \Phi^{-1}\left(y^{*}\right)-\operatorname{cl}_{\beta X} Z_{X}(f),
$$

there is a compact nbd $F$ of $z^{*}$ such that $F \cap \operatorname{cl}_{\beta_{X}} Z_{X}(f)=\phi$. Since $X$ is dense in $\beta X$, we have that $F \cap X \neq \phi$ and $f>\alpha$ on $F \cap X$ for some $\alpha>0$. This means that $f_{n}>\alpha_{n}$ on $F \cap X$ for some $\alpha_{n}>0$, i.e., $\operatorname{cl}_{\beta X} Z_{X}\left(f_{n}\right)$ does not contain $z^{*}$. This is a contradiction. Thus

$$
\Phi^{-1}\left(y^{*}\right) \subset \mathrm{cl}_{\beta X} Z_{X}(f) .
$$

Let $g$ be an extension of $f$ over $\beta X$, then it is obvious that

$$
\Phi^{-1}\left(y^{*}\right) \subset Z(g) \text {. }
$$

On the other hand, $Z(g)$ is not a nbd of $y^{*}$ because $\operatorname{cl}_{\beta X} Z_{X}(f)$ is not a nbd of $y^{*}$. Therefore the function $g$ is a desired function in (4).

$(4) \rightarrow(2)$. Let $y^{*}$ be any point in $\beta Y-Y$ and let $g$ be a function described in the assumption (4). Without loss of generality we can assume that $g \geqq 0$. Since $\Phi$ is open and closed by 4.4 and

$$
\Phi^{-1}\left(y^{*}\right) \subset Z_{\beta X}(g),
$$

$g^{s}$ is continuous on $\beta Y$ by 4.1 and $g^{s}\left(y^{*}\right)=0$. Since $Z_{\beta X}(g)$ is not a nbd of $\Phi^{-1}\left(y^{*}\right), \Phi\left(\beta X-Z_{\beta X}(g)\right)$ is open and does not contain $y^{*}$ but $\operatorname{cl}_{\beta Y} \Phi\left(\beta X-Z_{\beta X}(g)\right)$ contains $y^{*}$. By the method of the construction of $g^{s}$, we see that $g^{s}>0$ on $\Phi\left(\beta X-Z_{\beta_{X}}(g)\right)$ and hence

$$
Z_{\beta Y}\left(g^{s}\right) \subset \beta Y-\Phi\left(\beta X-Z_{\beta X}(g)\right) .
$$

Thus $Z_{\beta Y}\left(g^{s}\right)$ is not a nbd of $y^{*}$, that is, $y^{*}$ is not a $P$-point of $\beta Y$.

CoRollary 6.4. If $\varphi$ is an open WZ-mapping from a realcompact space $X$ onto a pseudocompact space $Y$, then $Y$ must be compact.

Proof. If $Y$ is not compact, then $\beta Y=v Y \neq Y$ and $Y_{c e} \cup Y_{c d}$ is compact by 6.2. $Z=\beta Y-Y_{c e}-Y_{c d}$ is an open locally compact subspace of $\beta Y$. Since every point $z$ of $Z-Y$ is a $P$-point of $\beta Y$ by $6.2, z$ has the compact nbd which is a $P$-space. On the other hand, a countably compact $P$-space is a finite set, and hence, $z$ must be isolated. This is a contradiction, since $z \in \beta Y-Y$.

Frolík [2] has proved the following

THEOREM $\left(F_{1}\right)$. The realcompactness is invariant under an open perfect mapping where $\varphi: X \rightarrow Y$ is said to be perfect if $\varphi$ is closed and compact. 
The following theorem is a generalization of Theorem $\left(F_{1}\right)$.

THEOREM 6.5. If $\phi$ is an open closed mapping from a realcompact space $X$ onto a space $Y$ such that $\mathscr{L} \phi^{-1}(y)$ is compact for every $y \in Y$ (equivalently $Y_{e}=\phi$ ), then $Y$ is also realcompact.

Proof. Since every $\mathscr{L} \varphi^{-1}(y)$ is compact, we have

$$
Y=Y_{c e} \cup Y_{c d} \cup Y_{d} \text { and } Y_{c e} \cup Y_{c d}
$$

is closed in $v Y$ by 6.2. If $y^{*} \in v Y-Y$, then $y^{*}$ is a $P$-point of $\beta Y$ by 6.2 , and hence there exists an open-closed nbd $W($ in $\beta Y)$ of $y^{*}$ with $V=W \cap Y \subset Y_{d}$. Let $x_{\alpha}$ be any point in $\varphi^{-1}\left(y_{\alpha}\right), y_{\alpha} \in V$, and $A=\left\{x_{\alpha}\right\}$. A is a discrete closed subset of $X$. Since $A$ is a closed subset of a realcompact space, $A$ is realcompact. $V$ is homeomorphic with $A$, and hence $V$ is realcompact. $V$ being open-closed, we have

$$
y^{*} \in U V \subset W \text {. }
$$

This contradicts $V=v V$. Thus $Y$ must be realcompact.

REMARK. It seems to me that Theorem 6.5 is not obtained directly from Theorem $\left(F_{1}\right)$ in the usual method below.

Let $\varphi$ be a mapping in 6.5. For $y \in Y_{c e}$ (notice $\left.Y_{e}=\phi\right)$,

$$
\varphi^{-1}(y)=\mathscr{L} \varphi^{-1}(y)
$$

and it is compact. For $y \in Y_{c d} \cup Y_{d}, \varphi^{-1}(y)$ is open-closed. We consider a subset $X_{0}=X_{c e} \cup X_{c d} \cup\left\{z ; z\right.$ is the point of $\left.\varphi^{-1}(y), y \in Y_{d}\right\}$. Then $X_{0}$ is a closed subset of $X$, and hence, it is realcompact. Let $\varphi_{0}$ be a mapping from $X_{0}$ onto $Y$ defined by $\varphi_{0}(x)=\varphi(x)$. It is obvious that $\varphi_{0}$ is a perfect mapping, but, from such a construction $\varphi_{0}$ is not in general necessarily open (if in this case, $\varphi_{0}$ is open, then 6.5 is an immediate consequence of Theorem $\left(F_{1}\right)$ ). For instance, let $N=\left\{t_{n}\right\}$ be the set of all natural numbers, $A_{n}=N, B_{n}=\beta A_{n}$ and let $C_{n}=B_{n}-A_{n}(n=1,2, \cdots)$. We denote by $M$ the topological sum of $A_{n}$. Then $B_{n} \subset \beta M$ and $B_{n}$ is open in $B M$. Let us put

$$
Z_{1}=Z_{2}=\beta M
$$

and we define a mapping $\psi_{i}$ from $Z_{i}$ onto $Y=\beta N$ by the Stone extension of the mapping $\lambda_{i}$ from $M$ onto $N$ with $\lambda_{i}\left(A_{n}\right)=t_{n}(i=1,2)$. Since $\lambda_{i}$ is open-closed, $\psi_{i}$ is also open-closed by 4.4 . Let $X$ be the topological sum of $Z_{1}-\cup C_{n}$ and $Z_{2}$ and define a mapping $\varphi$ from $X$ onto $Y$ by $\varphi\left|\left(Z_{1}-\cup C_{n}\right)=\psi_{1}\right|\left(Z_{1}-\cup C_{n}\right)$ and $\varphi \mid Z_{2}=\psi_{2}$. We shall prove the openness of $\varphi$. Since $\varphi^{\prime}=\varphi \mid\left(Z_{1}-\cup C_{n}\right)$ is a $W Z$-mapping 
from $Z_{1}-\cup C_{n}$ onto $Y$ and $\psi_{1}$ is an extension mapping of $\varphi^{\prime}$ from $\beta\left(Z_{1}-\cup C_{n}\right)=Z_{1}$ onto $Y$, we have by 4.4 that $\varphi^{\prime}$ is open. Thus it is easy to see that $\phi$ is open. Next we shall prove the closedness of $\varphi$. To do this, it is sufficient to show that $\varphi \mid\left(Z_{1}-\cup C_{n}\right)$ is closed. Let $F$ be a closed subset of $Z_{1}-\cup C_{n}$. Since $B_{n}$ is open in $Z_{1}$,

$$
\mathrm{cl}_{Z_{1}} F \cap B_{n} \neq \phi
$$

implies $F \cap A_{n} \neq \phi$. Thus we have $\psi_{1}\left(\mathrm{cl}_{Z_{1}} F\right)=\varphi(F)$, i.e., $\varphi$ is closed.

Let $a_{n}$ be the point of $A_{n} \subset Z_{1}(n=1,2, \cdots)$ and let $A=\left\{a_{n}\right\}$ and $X_{0}=\left(Z_{1}-\cup B_{n}\right) \cup \mathrm{cl}_{Z_{1}} A \cup\left(Z_{2}-\cup B_{n}\right)$ and $\varphi_{0}=\varphi \mid X_{0}$. Since $X_{0}$ is closed in $X, \varphi_{0}$ is a mapping considered in the begining of this remark. $U=X_{0}-\operatorname{cl}_{Z_{1}} A$ is open in $X_{0}$ but $\varphi_{0}(U)$ is contained in $Y-N$, and hence, $\varphi_{0}(U)$ is not open. This shows that $\varphi_{0}$ is not an open mapping.

By 6.5, it is proved that if $\varphi: X \rightarrow Y$ is an open $W Z$-mapping and if some condition imposed on $X$ implies $Y_{e}=\phi$, then $Y$ is realcompact when $X$ is realcompact. There exist many examples of such conditions. For instance, we have the following theorem.

THEOREM 6.6. Let $\varphi$ be an open WZ-mapping from a realcompact space $X$ onto $Y$. If $X$ is any one of the following spaces, then $Y$ is realcompact.

(1) $X$ is weakly separable.

(2) $X$ is locally compact.

(3) $X$ is connected.

(4) $X$ is locally connected.

(5) $X$ is perfectly normal.

7. Closed mappings and realcompactness. Frolík has proved the following:

THEOREM $\left(F_{2}\right)[3]^{2}$. If $\varphi$ is a perfect mapping from a realcompact, normal space $X$ onto $Y$, then $Y$ is realcompact.

In this section, we shall deal with closed mappings and show, in Theorem 7.5, that the realcompactness is invariant under a closed mapping, in Theorem $\left(F_{2}\right)$, if we replace "compactness of $\varphi$ " by "local compactness of $X$ ". It seems to me that Theorem 7.5 is only one case for which the realcompactness is proved to be invariant under a closed mapping without any additional condition.

LEMMA 7.1. If $\phi$ is a closed mapping from a normal space

${ }^{2}$ It seems to me that the countable paracompactness is necessary. 
$X$ onto $Y$, then $\operatorname{cl}_{\beta X} \mathscr{L}_{X} \varphi^{-1}(y)=\mathscr{L}_{\beta X} \Phi^{-1}(y)$ for every $y \in Y$. Furthermore, if $\mathscr{L}_{X} \phi^{-1}(y)$ is compact, then $\Phi^{-1}(y)-\varphi^{-1}(y)$ is open-closed in $\beta X-X$.

Proof. Since $\varphi$ is closed, we have $\operatorname{cl}_{\beta X} \varphi^{-1}(y)=\Phi^{-1}(y)$ by 1.1 and 1.2. It is obvious that $\mathscr{L}_{X} \varphi^{-1}(y) \subset \mathscr{L}_{\beta X} \Phi^{-1}(y)$. Suppose that there is a point $x$ in $\mathscr{L}_{\beta X} \Phi^{-1}(y)-\operatorname{cl}_{\beta X} \mathscr{L}_{X} \Phi^{-1}(y)$. We can find a nbd $U$ (in $\beta X)$ of $x$ with $\operatorname{cl}_{\beta X} U \cap \operatorname{cl}_{\beta X} \mathscr{L}_{X} \mathcal{P}^{-1}(y)=\phi$. Since

$$
\operatorname{cl}_{\beta X} \mathcal{P}^{-1}(y)=\Phi^{-1}(y), F=\operatorname{cl}_{\beta X} U \cap \varphi^{-1}(y) \neq \phi .
$$

Next we shall show that $E=\operatorname{cl}_{\beta X} U \cap\left(X-\varphi^{-1}(y)\right) \neq \phi$. Since

$$
x \in \mathscr{L}_{\beta_{X}} \Phi^{-1}(y)-\operatorname{cl}_{\beta X} \mathscr{L}_{X} \mathcal{P}^{-1}(y),
$$

$U$ contains a point $z$ of $\beta X-\Phi^{-1}(y)$, and hence, there is a nbd $V$ (in $\beta X$ ) of $z$ such that $V \subset U$ and $V \cap \Phi^{-1}(y)=\Phi$. $\quad X$ being dense in $\beta X, V$ contains a point of $X-\varphi^{-1}(y)$. Thus $E \neq \phi$. Since

$$
E \cap F=\operatorname{cl}_{\beta X} U \cap \varphi^{-1}(y) \cap\left(X-\varphi^{-1}(y)\right)=\phi
$$

and $X$ is normal, we have $\operatorname{cl}_{\beta X} E \cap \mathrm{cl}_{\beta X} F=\phi$. On the other hand, since $x \in \Phi^{-1}(y)=\operatorname{cl}_{\beta X} \mathcal{P}^{-1}(y)$ and $U$ is a nbd of $x$, we have $\operatorname{cl}_{\beta X} F \ni x$ and $\operatorname{cl}_{\beta X} E \ni x$, i.e., $\operatorname{cl}_{\beta X} F \cap \mathrm{cl}_{\beta X} E \neq \phi$ which is a contradiction. The latter part is obvious.

In the following, $Y_{c}=\left\{y ; y \in Y, \varphi^{-1}(y)\right.$ is compact $\}, Y_{0}=\{y ; y \in Y$, $\mathscr{L} \varphi^{-1}(y)$ is compact but $\varphi^{-1}(y)$ is not compact $\}$ and $Y_{1}=\{y ; y \in Y$, $\mathscr{L} \varphi^{-1}(y)$ is not compact\}.

THEOREM 7.2. ${ }^{3}$ Let $\varphi$ be a closed mapping from a locally compact, realcompact, normal space $X$ onto $Y$; then we have

(a) $Y_{0} \cup Y_{1}$ is closed.

(b) $Y-Y_{1}$ is locally compact.

(c) The closure of any neighborhood of $y$ is not compact for every $y \in Y_{1}$.

(d) $Y_{0} \cup Y_{1}$ is a discrete closed subset of $Y$.

Proof. (a). Let $y \in Y_{c}$ be an accumulation point of $Y_{0} \cup Y_{1}$. Since $\varphi^{-1}(y)$ is compact, there is a nbd $V$ of $\varphi^{-1}(y)$ whose closure is compact. $\quad M=Y-\varphi(X-V)$ is an open set containing $y$. Therefore there is a point $y^{\prime} \in Y_{0} \cup Y_{1}$ with $y^{\prime} \in M$. This shows that

$$
\varphi^{-1}\left(y^{\prime}\right) \subset \varphi^{-1}(M) \subset V \subset \bar{V}
$$

and $\varphi^{-1}\left(y^{\prime}\right)$ is compact. This is a contradiction.

3 This theorem is analogous to Theorem 4 in [11] in which $X$ is locally compact, paracompact, normal space. The proofs of (a) and (b) are the very same as those given in [11]. 
(b). Let $y$ be any point of $Y-Y_{1}$. Since $\mathscr{L} \varphi^{-1}(y)$ is compact, there is a nbd $V$ of $\mathscr{L} \varphi^{-1}(y)$ whose closure is compact.

$$
M=Y-\varphi(X-U)
$$

is an open set containing $y$ where $U=\varphi^{-1}(y) \cup V$. Then

$$
\bar{M} \subset \overline{\varphi(U)}=\varphi(\bar{U})=\varphi(\bar{V}) \cup\{y\}
$$

is compact, and hence, $\bar{M}$ is compact. This shows that $Y-Y_{1}$ is locally compact.

(c). Suppose that there is a point $y \in Y_{1}$ which has a nbd $W$ with the compact closure. Since $\mathscr{L}_{x} \varphi^{-1}(y)$ is not compact, there is a point $x \in \mathscr{L}_{X} \Phi^{-1}(y)-\mathscr{L}_{\beta X} \mathcal{P}^{-1}(y)$ by 7.1 , and hence there is a function $f \in C(\beta X)$ with $0 \leqq f \leqq 1, f(x)=0, f>0$ on $X$ by 1.8 since $X$ is realcompact. We shall show that there is a sequence $\left\{z_{n}\right\}$ in

$$
\varphi^{-1}(W)-\varphi^{-1}(y)
$$

such that $\varphi\left(z_{n}\right) \neq \varphi\left(z_{m}\right)(n \neq m)$ and $\left\{f\left(z_{n}\right)\right\} \downarrow 0$. For

$$
A_{n}=\left\{z ; f(z) \leqq 1 / n, z \in \varphi^{-1}(W)-\varphi^{-1}(y)\right\} \quad(n=1,2, \cdots),
$$

we have $x \in \operatorname{cl}_{\beta X} A_{n}$. If $\varphi\left(A_{n}\right)$ is finite, then $\varphi\left(A_{n}\right)$ does not contain $y$ since $\rho$ is closed. On the other hand, since $y \in \operatorname{cl}_{\beta X} A_{n}$ and $y \in Y$, we have $y \in \Phi\left(\operatorname{cl}_{\beta X} A_{n}\right) \subset \operatorname{cl}_{\beta Y} \Phi\left(A_{n}\right)=\operatorname{cl}_{\beta Y} \varphi\left(A_{n}\right)$, and hence, $y \in Y \cap \operatorname{cl}_{Y} \varphi\left(A_{n}\right)=\varphi\left(A_{n}\right)$. Thus every $A_{n}$ contains infinitely many points whose images, under $\varphi$, are distinct from each another. Therefore we have a desired sequence $\left\{z_{n} ; X_{n} \in A_{n}\right\}$ (if necessary, take a suitable subsequence). Since $f>0$ on $X, Z=\left\{z_{n}\right\}$ is a discrete closed subset. On the other hand, $\varphi(Z) \subset \bar{W}$ and $\bar{W}$ is compact, and hence, $\varphi(Z)$ has an accumulation point in $\varphi(Z)$. Let say $y_{0}=\varphi\left(z_{1}\right)$ be such an accumulation point because $\varphi$ is closed. $X$ being normal, there is an open set $U$ with $\varphi^{-1}\left(y_{0}\right) \subset U$ and $U \cap\left\{z_{n} ; n=2,3, \cdots\right\}=\phi$.

$$
M=Y-\varphi(X-U)
$$

is an open set containing $y_{0}$ which is disjoint from a closed set

$$
\varphi(Z)-\left\{y_{0}\right\}=\varphi\left(Z-\left\{z_{1}\right\}\right)
$$

because $Z-\left\{z_{1}\right\}$ is closed. This is a contradiction.

$(d)$. We shall prove that every point of $Y_{1}$ is isolated in $Y_{0} \cup Y_{1}$. If $\varphi^{-1}(y)$ has an open nbd $U$ such that $\varphi(U) \cap\left(Y_{0} \cup Y_{1}\right)=\{y\}$, then $M=Y-\varphi(X-U)$ is an open set with $\left(Y_{0} \cap Y_{1}\right) \cap M=\{y\}$. This shows that every point of $Y_{1}$ is isolated in $Y_{0} \cup Y_{1}$. Therefore, we can assume that there are a point $y \in Y_{1}$ and a point $x$ in $\varphi^{-1}(y)$ such that any open nbd $U$ of $x$ has a compact closure and $\varphi(U) \cap\left(Y_{0} \cup Y_{1}\right)$ 
contains infinitely many points $y_{n}(n=1,2, \cdots)$ of $Y_{0} \cup Y_{1}$. Let $a_{n}$ be any point contained in $\varphi^{-1}\left(y_{n}\right) \cap U$. Then $\left\{a_{n}\right\}$ has an accumulation point $a_{0}$ in $\bar{U}$ because $\bar{U}$ is compact. Since $\varphi\left(a_{n}\right)=y_{n} \in Y_{0} \cup Y_{1}$ and $Y_{0} \cup Y_{1}$ is closed by (a), we have $y_{0}=\varphi\left(a_{0}\right) \in Y_{0} \cup Y_{1}$. Thus we can assume that there is a point $y_{0} \in Y_{0} \cup Y_{1}$ which is an accumulation point of $\left\{y_{n} ; y_{n} \in Y_{0} \cup Y_{1}\right\}$. Let $x_{n}^{\prime} \in \Phi^{-1}\left(y_{n}\right)-\varphi^{-1}\left(y_{n}\right)$; then $\beta X-X$ being compact, $A \cap X=\phi$ where $A=\operatorname{cl}_{\beta X}\left\{x_{n}^{\prime}\right\}$. If $A \cap \Phi^{-1}\left(y_{0}\right)=\phi$, then $y_{0} \notin \Phi(A)$ which is impossible because $y_{n} \in \Phi(A)(n=1,2, \cdots)$ and $\Phi$ is closed. Let $x_{n}^{\prime} \in A \cap\left(\Phi^{-1}\left(y_{0}\right)-\phi^{-1}\left(y_{0}\right)\right)$ and $f$ be a function of $C(\beta X)$ such that $0 \leqq f \leqq 1, f\left(x_{0}^{\prime}\right)=0$ and $f>0$ on $X$ by 1.8 because $X$ is realcompact. Since $\operatorname{cl}_{\beta X} \varphi^{-1}(y)=\Phi^{-1}(y)$, without loss of generality, we can find a point $x_{n}$ in $U_{n} \cap \varphi^{-1}\left(y_{n}\right)$ for every $n$ such that $\left\{f\left(x_{n}\right)\right\} \downarrow 0$ where $U_{n}$ is an open nbd (in $\beta X$ ) of $x_{n}^{\prime}$. If $B \cap \varphi^{-1}\left(y_{0}\right)=\phi$ where $B=\operatorname{cl}_{X}\left\{x_{n} ; n=1,2, \cdots\right\}$, then $\varphi(B)=\varphi(\bar{B})=\overline{\varphi(B)}=\overline{\left\{y_{n}\right\}}$ does not contain $y_{0}$. This is impossible. Thus $B \cap \varphi^{-1}\left(y_{0}\right)$ contains a point $x_{0}$. It is obvious that $f\left(x_{0}\right)=0$, but, this is a contradiction because $f>0$ on $X$. Thus every point of $Y_{1}$ is isolated in $Y_{0} \cup Y_{1}$.

Next we shall prove that every point $y$ of $Y_{0}$ is isolated in $Y_{0} \cup Y_{1}$, which shows that $Y_{0} \cup Y_{1}$ is a discrete closed subset of $Y$.

$$
\Phi_{1}=\Phi \mid(\beta X-X)
$$

is a closed mapping from a compact space $\beta X-X$ onto $\beta Y-Y_{c}$. For every $y \in Y_{0}, \Phi^{-1}(y)-\varphi^{-1}(y)$ is always open-closed by 7.1 in $\beta X-X$. Thus every point of $Y_{0}$ is isolated in $\beta Y-Y_{c}$, and hence, they are isolated in $Y_{0} \cup Y_{1}\left(\subset \beta Y-Y_{c}\right)$.

From (b) and (c) in 7.2, we have:

THEOREM 7.3. Let $\varphi$ be a closed mapping from a locally compact, realcompact, normal space $X$ onto $Y$; then $Y$ is locally compact if and only if $\mathscr{L} \varphi^{-1}(y)$ is compact for every $y \in Y$.

This theorem is not necessarily true in general when $X$ is locally compact normal, as shown by the following example by Prof. Morita. Let $X=[0,1] \times W\left(\omega_{1}\right), Y=[0,1]$ and let $\varphi$ be the projection: $X \rightarrow Y$. It is known that $X$ and $Y$ are both locally compact normal. Since $Y$ is weakly separable and $X$ is countably compact, $\varphi$ is closed, but $\varphi^{-1}(\alpha)$ is not compact for every $\alpha \in Y$. Theorem 7.3 is also true, as shown in [11] replacing "realcompactness" by "paracompactness".

Under the assumption of 7.2 , we shall consider the new space $Z$ in the following way: we set up an equivalence relation " " on $X$ by the simple rule that " $x \sim x^{\prime \prime}$ " if and only if both points $x$ and $x^{\prime}$ belongs to the same $\varphi^{-1}(y)$ for some point $y \in Y_{0} \cup Y_{1}$. Using this relation we define a space $Z$, that is, $Z$ is a space obtained from $X$ 
by the topological identification (we notice that $V$ of $Z$ is open if and only if $\psi^{-1}(V)$ is open where $\psi$ is the identification mapping). It is easy to see that $Z_{c}=\psi\left(X_{c}\right)$ is locally compact and homeomorphic with $X_{c}$, and $Z_{0} \cup Z_{1}$ is a discrete closed subset where

$$
X_{c}=\varphi^{-1}\left(Y_{c}\right), X_{i}=\varphi^{-1}\left(Y_{i}\right)(i=0,1), Z_{0}=\psi\left(X_{0}\right)
$$

and $Z_{1}=\psi\left(X_{1}\right) . \quad \psi$ is obviously closed, and hence, $Z$ is normal.

Now suppose that $X$ is realcompact. $Z_{0} \cup Z_{1}$ is realcompact as in the proof of realcompactness of $V$ in 6.5 since $Z_{0} \cup Z_{1}$ is closed and discrete. If every function of $C(Z)$ is continuously extended over a point $z$ in $\beta Z-Z$, then there is a nbd $U($ in $\beta Z)$ with $\operatorname{cl}_{\beta Z} U \cap\left(Z_{0} \cup Z_{1}\right)=\phi$ because $Z_{0} \cup Z_{1}$ is closed and realcompact. Thus $\operatorname{cl}_{\beta Z} U \cap Z_{c} \neq \phi$, but this is impossible since $Z_{c}$ is homeomorphic with $X_{c}$. Therefore $Z$ becomes a realcompact space.

Next we can construct a mapping $\lambda$ from $Z$ onto $Y$ by the usual topological identification and it is easily seen that $\lambda$ is perfect. Thus we have.

CoRollary 7.4. Let $\varphi$ be a closed mapping from a realcompact, locally compact, normal space $X$ onto $Y$; then $\varphi$ admits a factorization $\varphi=\lambda \psi$ such that

(i) \& is a closed mapping from $X$ onto a realcompact normal space $Z$ and $\left\{\psi^{-1}(z) ; z \in Z^{\prime}\right\}$ is a closed discrete collection where $Z^{\prime}$ is the set of point $z$ such that $\psi^{-1}(z)$ contains at least two points.

(ii) $\lambda: Z \rightarrow Y$ is a perfect mapping.

Since countable paracompactness is invariant under a closed mapping, we have the following theorem by 7.2 and Theorem $\left(F_{2}\right)$.

Theorem 7.5. If $\phi$ is a closed mapping from a locally compact, countably paracompact, normal space $X$ onto $Y$, then $Y$ is realcompact when $X$ is realcompact.

8. Examples. Let $M$ be a $P$-space and let $K$ be a separable metric space. We denote by $\rho$ the projection: $M \times K \rightarrow M$ and by $\Phi$ the Stone extension of $\varphi$ from $\beta(M \times K)$ onto $\beta M$. Next $\psi$ denotes the identity mapping on $M \times K$ and $\Psi$ denotes the extension of $\psi$ from $\beta(M \times K)$ onto $\beta M \times \beta K$ and let $\Psi_{0}=\Psi \mid Z$ where

$$
Z=\cup\left\{\Phi^{-1}(y) ; y \in M\right\} \subset \beta(M \times K) .
$$

Lemma 8.1. (1) The projection $\varphi: M \times K \rightarrow M$ is closed.

(2) $Z$ is realcompact if $M$ is realcompact.

(3) $\Psi_{0}$ is a one-to-one mapping from $Z$ onto $M \times \beta K$. 


\section{(4) $\Psi^{-1}(M \times \beta K)=Z$.}

Proof. (1). Let $F$ be a closed subset of $M \times K$ and let $y \notin \varphi(F)$. Now suppose that $y$ is not isolated. Since $F$ is closed, for a point $(y, z) \in \varphi^{-1}(y)$, there is a nbd $W(y, z)=V(y) \times U(z)$ of $(y, z)$ such that $W(y, z) \cap F=\phi$, where $V(y)$ and $U(z)$ are neighborhoods of $y$ and $z$ in $M$ and $K$ respectively. Since $K$ is separable and $\{W(y, z) ; z \in K\}$ covers $\varphi^{-1}(y)$, there is a subcover $\left\{W\left(y, z_{i}\right) ; i=1,2, \cdots\right\}$. Let us put $V=\cap V_{i}$; then $V$ is a nbd of $y$ because $y$ is a $P$-point, and hence, $V \times K$ is open and $(V \times K) \cap F=\phi$. This implies $y \notin \varphi(F)$ since $\varphi^{-1}(y) \subset V \times K$. Thus $\varphi(F)$ is a closed subset which shows the closedness of $\varphi$. by 5.3 .

(2). Since $\Phi$ is closed and $\bar{\Phi}^{-1}(y)$ is compact, $Z$ is realcompact

(3) Since $\varphi$ is closed, $\Phi^{-1}(y)=\operatorname{cl}_{\beta(\mu \times K)} \varphi^{-1}(y)$, and

$$
\psi \varphi^{-1}(y) \subset \Psi_{0}\left(\Phi^{-1}(y)\right) \text {. }
$$

On the other hand, $\psi^{\prime} \varphi^{-1}(y)=\{y\} \times K$ is dense in $\{y\} \times \beta K$. This implies that $\Psi_{0}\left(\Phi^{-1}(y)\right)=\{y\} \times \beta K$, equivalently $\Psi_{0}^{-1}(\{y\} \times \beta K)=\Phi^{-1}(y)$ because $\Phi^{-1}(y)$ is compact. Thus $\Psi_{0}(Z)=M \times \beta K$, that is, $\Psi_{0}$ is onto.

Next we shall show that $\Psi_{0}$ is one-to-one. Suppose that there are a point $y^{*} \in(\{y\} \times \beta K)-(\{y\} \times K)$ and $x_{1}, x_{2} \in \Psi_{0}^{-1}\left(y^{*}\right), x_{1} \neq x_{2}$. There are open sets $V_{1}($ in $Z)$ and $V_{2}($ in $Z)$ of $x_{1}$ and $x_{2}$ respectively with $\bar{V}_{1} \cap \bar{V}_{2}=\phi$. Let us put $F_{i}=\bar{V}_{i} \cap \varphi^{-1}(y)$; then $F_{i} \neq \phi$ since $\Phi^{-1}(y)=\operatorname{cl}_{\beta(M \times K)} \varphi^{-1}(y)$. Since $\operatorname{cl}_{M \times \beta K} \psi\left(F_{i}\right) \subset\{y\} \times \beta K, \quad F_{i}$ is a closed subset of a normal space $\{y\} \times K$ and $\beta(\{y\} \times K)=\{y\} \times \beta K$, we have $\mathrm{cl}_{M \times \beta K} \psi\left(F_{1}\right) \cap \mathrm{cl}_{M \times \beta K} \psi\left(F_{2}\right)=\phi$. On the other hand,

$$
x_{i} \in \operatorname{cl}_{\beta(\boldsymbol{M} \times \boldsymbol{K}) \cap Z} F_{i} \subset \Phi^{-1}(y)
$$

implies that $y^{*} \in \Psi_{0}\left(\operatorname{cl}_{\beta(M \times K) \cap Z} F_{i} \subset \operatorname{cl}_{M \times \beta K} \Psi_{0}\left(F_{i}\right)=\operatorname{cl}_{M \times \beta K} \psi\left(F_{i}\right) \quad(\mathrm{i}=1,2)\right.$. This is a contradiction.

(4). Suppose that there is a point $w \in \beta(M \times K)-Z$ such that $\Psi(w)=(y, \alpha) \in M \times \beta K$. There are open subsets $V_{1}$ and $V_{2}$ in $\beta(M \times K)$ such that $w \in V_{2}, \Phi^{-1}(y) \subset V_{1}$ and $\bar{V}_{1} \cap \bar{V}_{2}=\phi$. $\quad \bar{V}_{2} \cap Z$ is not empty and $\Phi\left(\bar{V}_{2} \cap Z\right)$ is a subset of $M$ containing $y$. Since

$$
\Psi_{0}^{-1}(\{y\} \times \beta K)=\Phi^{-1}(y)
$$

by (3), we have $\Psi_{0}\left(\bar{V}_{2} \cap Z\right) \cap(\{y\} \times \beta K)=\phi$. Let $\mu$ be the projection: $M \times \beta K \rightarrow M$; then, we have $\Phi(A)=\mu \Phi_{0}(A)$ for every subset $A$ of $Z$ because $\Psi_{0}^{-1}(\{y\} \times \beta K)=\Phi^{-1}(y)$. Thus

$$
\Phi\left(\bar{V}_{2} \cap Z\right)=\mu \Psi_{0}\left(\bar{V}_{2} \cap Z\right) \nexists y
$$

which is a contradiction, and hence, we have $Z=\Psi^{-1}(M \times \beta K)$. 
Let $M$ be a realcompact nondiscrete $P$-space; then $M \times K$ is realcompact and there is a function $f$ of $C(\beta(M \times K)$ ) such that $f>0$ on $M \times K$ and $Z(f) \cap \Phi^{-1}(y) \neq \phi$ for a given nonisolated point $y$ of $M$. We notice $\Phi^{-1}(y) \operatorname{cl}_{\beta(M \times K)} \varphi^{-1}(y)=\operatorname{cl}_{z} \varphi^{-1}(y)(\cong\{y\} \times \beta K)$. In the following, we put $A_{y}=\Phi^{-1}(y)$ and $B_{y}=A_{y}-\varphi^{-1}(y)$.

Next we shall show that we cannot replace a $Z$-mapping by an open WZ-mapping in Theorem 5.3.

EXAMPLE 8.2. $X=Z-\left(Z(f) \cap B_{y}\right)$ is not realcompact and a mapping $\lambda=\Phi \mid X$ is an open $W Z$-mapping from $X$ onto $M$ and $\lambda$ is not a $Z$-mapping.

Proof. It is obvious that $\Phi$ is open and closed, $X$ is open in $Z$, $\Phi^{-1}\left(y^{\prime}\right)=\lambda^{-1}\left(y^{\prime}\right)$ for every $y^{\prime}(\neq y)$ and

$$
\left.\left.\varphi^{-1}(y) \sqsubset \lambda^{-1}(y)=A_{y}-Z(f) \cap B_{y}\right)\right),
$$

and hence $\lambda$ is an open $W Z$-mapping. Thus to prove 8.2 , it is sufficient to show that $X$ is not realcompact by 5.3. Suppose that $X$ is realcompact, then there are a function $h \in C(X)$ and a point $x^{*} \in B_{y}$ such that $h$ can not be continuously extended over $x^{*}$. Since every subset $\lambda^{-1}\left(y^{\prime}\right)=\Phi^{-1}\left(y^{\prime}\right)$ is compact for $y^{\prime} \neq y, h$ is bounded on $\lambda^{-1}\left(y^{\prime}\right)$. If $h$ is bounded on a $W \cap X$ where $W$ is a nbd (in $\beta(M \times K)$ ) of $x^{*}$, then $h$ is continuously extended over $x^{*}$. Thus for every nbd $W$ of $x^{*}, h$ is not bounded on $W \cap X$. Without loss of generality, we can assume that $h$ is nonnegative on $X$. Therefore, for every $n$, there is a nbd $W_{n}$ (in $\beta(M \times K)$ ) of $x^{*}$ with $h \geqq n$ on $W_{n} \cap X$. $\varphi^{-1}(y) \cap W_{n}$ contains a point $\left(y, k_{n}\right)$, and hence there are neighborhoods $O_{n}$ and $Q_{n}$ of $y$ and $k_{n}$ respectively such that $h \geqq n$ on $O_{n} \times Q_{n}$. Since $y$ is a $P$-point, $V=\cap O_{n}$ is a nbd of $y$ and $h$ is not bounded on

$$
A=\left\{\left(y_{0}, k_{n}\right) ; n=1,2, \cdots\right\}
$$

where $y_{0}$ is some point of $V$ and $y \neq y_{0}$. On the other hand, $h$ is bounded on $A$ and $A \subset \Phi^{-1}\left(y_{0}\right)$. This is a contradiction. Thus $X$ is not realcompact.

\section{BIBLIOGRAPHY}

1. A. Archangelski and A. Taimanov, On the theorem of $V$. Ponomarev, Deklady Acad. Nauk 135, (1960), 247-248.

2. Z. Frolík, Applications of complete family of continuous functions to the theory of Q-spaces, Czech. Math. Journ. 11 (89) (1961), 115-133.

3. Z. Frolík, On almost realcompact spaces, Bull. Acad. Polon. Sci. Sér. Sci. Math. Astronom Phys. IX (1961), 247-250.

4. L. Gillman and M. Jerison, Rings of Continuous Functions, Van Norstrand, Princeton, N. J., 1960. 
5. S. Hanai, Inverse images of closed mappings $I, I I, I I I$, Proc. Japan Acad. 37 (1961), 298-301, 302-304, 457-458.

6. S. Hanai and A. Okuyama, On pseudocompactness and continuous mappings, Proc. Japan Acad. 38 (1962), 444-447.

7. E. Hewitt, Rings of real-valued continuous functions I, Trans. Amer. Math. Soc. 64 (1948), 45-99.

8. T. Isiwata, Some characterizations of countably compact spaces, Sci. Reports of Tokyo Kyoiku Daigaku, Ser. A., 5 (1956), 185-189.

9. T. Isiwata, On subspaces of $\check{C}$ ech compactification space, Sci. Reports of Tokyo Kyoiku Daigaku, Ser A., 5 (1957), 304-309.

10. S. Kasahara, Boundedness of semi-continuous finite real functions, Proc. Japan Acad. 33 (1957), 183-186.

11. K. Morita, On closed mappings, Proc. Japan Acad. 32 (1956), 539-543.

12. S. Mrówka, Compactness and product spaces, Colloquim Math. 7 (1959), 12-22.

Received July 17, 1964.

TOKYo GAKUGEI UNIVERSITY 


\title{
PACIFIC JOURNAL OF MATHEMATICS
}

\author{
EDITORS
}

H. Samelson

Stanford University

Stanford, California

J. P. JANS

University of Washington

Seattle, Washington 98105

\section{J. DugundJI}

University of Southern California Los Angeles, California 90007

RICHARD ARENS

University of California

Los Angeles, California 90024

\section{ASSOCIATE EDITORS}

E. F. BECKENBACH

B. H. NeUmanN

F. WOLF

K. YosIDA

\section{SUPPORTING INSTITUTIONS}

UNIVERSITY OF BRITISH COLUMBIA

CALIFORNIA INSTITUTE OF TECHNOLOGY

UNIVERSITY OF CALIFORNIA

MONTANA STATE UNIVERSITY

UNIVERSITY OF NEVADA

NEW MEXICO STATE UNIVERSITY

OREGON STATE UNIVERSITY

UNIVERSITY OF OREGON

OSAKA UNIVERSITY

UNIVERSITY OF SOUTHERN CALIFORNIA

\author{
STANFORD UNIVERSITY \\ UNIVERSITY OF TOKYO \\ UNIVERSITY OF UTAH \\ WASHINGTON STATE UNIVERSITY \\ UNIVERSITY OF WASHINGTON \\ * \\ AMERICAN MATHEMATICAL SOCIETY \\ CHEVRON RESEARCH CORPORATION \\ TRW SYSTEMS \\ NAVAL ORDNANCE TEST STATION
}

Mathematical papers intended for publication in the Pacific Journal of Mathematics should be typewritten (double spaced). The first paragraph or two must be capable of being used separately as a synopsis of the entire paper. It should not contain references to the bibliography. Manuscripts may be sent to any one of the four editors. All other communications to the editors should be addressed to the managing editor, Richard Arens at the University of California, Los Angeles, California 90024.

50 reprints per author of each article are furnished free of charge; additional copies may be obtained at cost in multiples of 50 .

The Pacific Journal of Mathematics is published monthly. Effective with Volume 16 the price per volume (3 numbers) is $\$ 8.00$; single issues, $\$ 3.00$. Special price for current issues to individual faculty members of supporting institutions and to individual members of the American Mathematical Society: $\$ 4.00$ per volume; single issues $\$ 1.50$. Back numbers are available.

Subscriptions, orders for back numbers, and changes of address should be sent to Pacific Journal of Mathematics, 103 Highland Boulevard, Berkeley 8, California.

Printed at Kokusai Bunken Insatsusha (International Academic Printing Co., Ltd.), No. 6, 2-chome, Fujimi-cho, Chiyoda-ku, Tokyo, Japan.

PUBLISHED BY PACIFIC JOURNAL OF MATHEMATICS, A NON-PROFIT CORPORATION

The Supporting Institutions listed above contribute to the cost of publication of this Journal, but they are not owners or publishers and have no responsibility for its content or policies. 


\section{Pacific Journal of Mathematics}

\section{Vol. 20, No. $3 \quad$ November, 1967}

Dallas O. Banks, Lower bounds for the eigenvalues of a vibrating string whose density satisfies a Lipschitz condition ................... 393

Ralph Joseph Bean, Decompositions of $E^{3}$ which yield $E^{3} \ldots \ldots \ldots \ldots 411$

Robert Bruce Brown, On generalized Cayley-Dickson algebras ......... 415

Richard Dowell Byrd, Complete distributivity in lattice-ordered groups . . . 423

Roger Countryman, On the characterization of compact Hausdorff $X$ for

which $C(X)$ is algebraically closed ...................... 433

Cecil Craig, Jr. and A. J. Macintyre, Inequalities for functions regular and bounded in a circle ...................................... 449

Takesi Isiwata, Mappings and spaces ..................... 455

David Lewis Outcalt, Power-associative algebras in which every subalgebra is an ideal ..................................... 481

Sidney Charles Port, Equilibrium systems of stable processes .......... 487

Jack Segal, Quasi dimension type. I. Types in the real line ............ 501

Robert William Stringall, Endomorphism rings of primary abelian

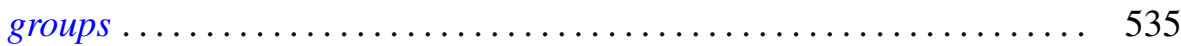

William John Sweeney, "The $\delta$-Poincaré estimate” ................. 559

L. Tzafriri, Operators commuting with Boolean algebras of projections of finite multiplicity ............................... 571 